\title{
Left-right asymmetries in polarized lepton-lepton scattering
}

\author{
J. C. Montero, V. Pleitez, and M. C. Rodriguez \\ Instituto de Física Teórica, Universidade Estadual Paulista, Rua Pamplona, 145, 01405-900 São Paulo, SP, Brazil
}

(Received 12 February 1998; published 2 October 1998)

\begin{abstract}
Several parity-violating left-right asymmetries in Møller electron-electron and muon-muon scattering are considered in the context of the electroweak standard model at the tree level in fixed target and collider experiments. We show that in colliders the asymmetry with only one of the beams polarized is large enough to compensate the smaller cross section at high energies. We also show that these asymmetries are very sensitive to a doubly charged vector bilepton resonance but they are insensitive to scalar ones. [S0556-2821(98)09219-4]
\end{abstract}

PACS number(s): 13.88.+e, 12.60.Cn

\section{INTRODUCTION}

It is well known that the so-called Next Linear Collider (NLC) will provide opportunities for both discovery and precision measurements [1]. With the construction of the next generation of $e^{+} e^{-}$linear colliders, with a center of mass energy up to $1500 \mathrm{GeV}$ and which will be able to operate also in $\gamma \gamma, \gamma e^{-}$, and $e^{-} e^{-}$modes, new perspectives arise in detecting new physics beyond the electroweak standard model in processes having nonzero initial electric charge (and nonzero lepton number). For instance, new resonances of doubly charged scalar and vector bosons will enhance the cross section and in this case it is possible that the measurement of the left-right asymmetry $\left(A_{R L}\right)$ in electron-electron scattering can be a better parameter to signal out those resonances (if any) than the total cross section itself. On the other hand, although the First Muon Collider (FMC) will be one of the type $\mu^{-} \mu^{+}$(it is a circular machine) [2], if this sort of technology were mastered, perhaps a Linear Muon Collider (LMC) will be feasible. In this work we will consider mainly $e^{-} e^{-}$processes but we will also comment on the muon case.

\section{A. Lepton Mbller scattering at high energies}

The appealing features for studying parity-violating asymmetries between the scattering of left- and right-handed polarized electrons on a variety of targets were pointed out some years ago by Derman and Marciano [3]. In particular, in the context of the electroweak standard model (ESM) [4] the measurement of the $A_{R L}$ asymmetry determined the relative sign between the weak and electromagnetic interactions.

It is well known that the left-right asymmetry in electronelectron Møller scattering (fixed target) and an unpolarized target is rather small, $\sim 10^{-7}$ (see below) [3]. Notwithstanding, since the scattering has a large cross section, we can expect that fixed target experiments like those at SLAC [5] will have a number of events enough to determine such a small number. The typical beam energy of such experiments is $50 \mathrm{GeV}$ and both the incident electron beam and the target electron can be polarized. On the other hand, collider experiments were stopped years ago at very low energies, around $1.2 \mathrm{GeV}$ [6]. Although the $A_{R L}$ asymmetry is larger in colliders than in fixed target experiments, the former have a smaller cross section. Hence, it seems at first sight that a measurement of that asymmetry with large statistics in colliders will be difficult.

In general we have processes such as

$$
l_{1}\left(p_{1}, \lambda_{1}\right)+l_{2}\left(p_{2}, \lambda_{2}\right) \rightarrow l_{1}^{\prime}\left(q_{1}, \Lambda_{1}\right)+l_{2}^{\prime}\left(p_{2}^{\prime}, \Lambda_{2}\right),
$$

when $l_{1,2}=e, \mu$ and $l_{1,2}^{\prime}=e, \mu, \tau, L$, with $L$ an exotic lepton. In Eq. (1), $p_{i}, q_{i}\left(\lambda_{i}, \Lambda_{i}\right)$ denote the initial and final lepton momenta (helicities). In this work we will consider only processes such as

$$
l\left(p_{1}, \lambda_{1}\right)+l\left(p_{2}, \lambda_{2}\right) \rightarrow l\left(q_{1}, \Lambda_{1}\right)+l\left(q_{2}, \Lambda_{2}\right)
$$

Note that if we were considering quarks in the final state, there must be a mixing of ordinary quarks with exotic ones since with the known quarks we cannot build a doubly charged state.

Our main goal is to compare left-right asymmetry in the context of the electroweak standard model in fixed target experiments with the respective one in colliders experiments. Since we are not neglecting the masses of the fermions, we can use the same amplitudes for the case of $e^{-} e^{-}$and $\mu^{-} \mu^{-}$scattering. We also study the behavior of that quantity when a vector or scalar bilepton is considered in collider experiments.

\section{B. Four-spinor helicity eigenstates and left-right asymmetry}

In this work we will not neglect the ESM Higgs-lepton interactions because in collider experiments with muons the Higgs contributions are not, in principle, negligible. Besides, in the future we will consider processes in which the final lepton can be $\tau$ or even an exotic lepton $L^{-}$(or even exotic quarks $J$ ). Hence, we will work with massive four-spinor eigenvectors of the helicity operator, defined as

$$
\Sigma=\left(\begin{array}{cc}
\vec{\sigma} \cdot \vec{p} /|\vec{p}| & 0 \\
0 & \vec{\sigma} \cdot \vec{p} /|\vec{p}|
\end{array}\right),
$$

with the gamma matrices in the chiral representation, which are given by 


$$
\begin{gathered}
u_{l R}=N_{l}\left(\begin{array}{c}
\left(1+\eta_{l}\right) \cos \left(\theta_{l} / 2\right) e^{-i \phi_{l} / 2} \\
\left(1+\eta_{l}\right) \sin \left(\theta_{l} / 2\right) e^{i \phi_{l} / 2} \\
\left(-1+\eta_{l}\right) \cos \left(\theta_{l} / 2\right) e^{-i \phi_{l} / 2} \\
\left(-1+\eta_{l}\right) \sin \left(\theta_{l} / 2\right) e^{i \phi_{l} / 2}
\end{array}\right), \\
u_{l L}=N_{l}\left(\begin{array}{c}
\left(-1+\eta_{l}\right) \sin \left(\theta_{l} / 2\right) e^{-i \phi_{l} / 2} \\
-\left(-1+\eta_{l}\right) \cos \left(\theta_{l} / 2\right) e^{i \phi_{l} / 2} \\
\left(1+\eta_{l}\right) \sin \left(\theta_{l} / 2\right) e^{-i \phi_{l} / 2} \\
-\left(1+\eta_{l}\right) \cos \left(\theta_{l} / 2\right) e^{i \phi_{l} / 2}
\end{array}\right),
\end{gathered}
$$

where we have defined

$$
N_{l}=\sqrt{\frac{E_{l}}{\left(1+\eta_{l}^{2}\right)}}, \quad \eta_{l}^{2}=\frac{E_{l}+m_{l}}{E_{l}-m_{l}},
$$

$E_{l}$ and $m_{l}$ being the total energy and rest mass for the lepton l. ( $\hbar=1$ and $c=1$ throughout this work.) In Eq. (4), $u_{R}$ and $u_{L}$ denote positive energy spinors with positive (right) and negative (left) helicity, respectively. Similarly expressions can be obtained for the negative energy solutions but we will not write them explicitly.

The left-right asymmetry is defined as

$$
A_{R L}(l l \rightarrow l l)=\frac{d \sigma_{R}-d \sigma_{L}}{d \sigma_{R}+d \sigma_{L}}
$$

where $d \sigma_{R(L)}$ is the differential cross section for one right(left-) handed lepton $l$ scattering on an unpolarized lepton $l$. That is,

$$
A_{R L}(l l \rightarrow l l)=\frac{\left(d \sigma_{R R}+d \sigma_{R L}\right)-\left(d \sigma_{L L}+d \sigma_{L R}\right)}{\left(d \sigma_{R R}+d \sigma_{R L}\right)+\left(d \sigma_{L L}+d \sigma_{L R}\right)},
$$

where $d \sigma_{i j}$ denotes the cross section for incoming leptons with helicity $i$ and $j$, respectively, and they are given by

$$
d \sigma_{i j} \propto \sum_{k l}\left|M_{i j ; k l}\right|^{2}, \quad i, j ; k, l=L, R .
$$

When both leptons are identical we can use the property $M_{R L ; R L}=M_{L R ; L R}$ (which implies $d \sigma_{R L}=d \sigma_{L R}$ ) assured by rotational invariance in the former case. Hence, for calculating the asymmetry $A_{R L}$ in Eq. (7) we use

$$
\begin{aligned}
& d \sigma_{R R} \propto\left|M_{R R ; R R}\right|^{2}+\left|M_{R R ; L R}\right|^{2}+\left|M_{R R ; R L}\right|^{2}+\left|M_{R R ; L L}\right|^{2}, \\
& d \sigma_{R L} \propto\left|M_{R L ; R R}\right|^{2}+\left|M_{R L ; R L}\right|^{2}+\left|M_{R L ; L R}\right|^{2}+\left|M_{R L ; L L}\right|^{2}, \\
& d \sigma_{L R} \propto\left|M_{L R ; R R}\right|^{2}+\left|M_{L R ; R L}\right|^{2}+\left|M_{L R ; L R}\right|^{2}+\left|M_{L R ; L L}\right|^{2},
\end{aligned}
$$

and

$$
d \sigma_{L L} \propto\left|M_{L L ; R R}\right|^{2}+\left|M_{L L ; R L}\right|^{2}+\left|M_{L L ; L R}\right|^{2}+\left|M_{L L ; L L}\right|^{2} .
$$

Another interesting possibility is the case when both leptons are polarized. We can define an asymmetry $A_{R ; R L}$ in which one beam is always in the same polarization state, say, right handed, and the other is either right- or left-handed polarized (similarly we can define $A_{L ; L R}$ ):

$$
A_{R ; R L}=\frac{d \sigma_{R R}-d \sigma_{R L}}{d \sigma_{R R}+d \sigma_{R L}}, \quad A_{L ; R L}=\frac{d \sigma_{L R}-d \sigma_{L L}}{d \sigma_{L L}+d \sigma_{L R}}
$$

We can define also an asymmetry when one incident particle is right handed and the other is left handed and the final states are right and left or left and right handed:

$$
A_{R L ; R L, L R}=\frac{d \sigma_{R L ; R L}-d \sigma_{R L ; L R}}{d \sigma_{R L ; R L}+d \sigma_{R L ; L R}}
$$

or, similarly, $A_{L R ; R L, L R}$. These asymmetries, in Eqs. (10) and (11), are also dominated by QED contributions. However, this will not be the case if a bilepton resonance exists at typical energies of the NLC. To show this fact is the goal of the next section. These asymmetries can be calculated for both fixed target (FT) and colliders (CO) experiments.

This work is organized as follows. In Sec. II we study the left-right asymmetries for both fixed target and collider experiments in the context of the ESM and for $e^{-} e^{-}$ $\rightarrow e^{-} e^{-}$. We also briefly consider the case of $\mu^{-} \mu^{-}$colliders. The same asymmetries for colliders are shown in Sec. III but considering the contributions of vector and scalar bileptons. Our conclusions appear in the last section while we left Appendixes A-E for showing the several amplitudes and cross sections we have used in this work.

\section{LEFT-RIGHT ASYMMETRIES IN THE ELECTROWEAK STANDARD MODEL}

Let us consider the lepton-lepton (diagonal) process in the context of the electroweak standard model at the tree level. The relevant part of the Lagrangian of this model is

$$
\begin{aligned}
\mathcal{L}_{F}= & -\sum_{i} \frac{g m_{i}}{2 M_{W}} \bar{\psi}_{i} \psi_{i} H^{0}-e \sum_{i} q_{i} \bar{\psi}_{i} \gamma^{\mu} \psi_{i} A_{\mu} \\
& -\frac{g}{2 \cos \theta_{W}} \psi_{i} \gamma^{\mu}\left(g_{V}^{i}-g_{A}^{i} \gamma^{5}\right) \psi_{i} Z_{\mu} .
\end{aligned}
$$

$\theta_{W} \equiv \tan ^{-1}\left(g^{\prime} / g\right)$ is the weak mixing angle, and $e=g \sin \theta_{W}$ is the positron electric charge with $g$ such that

$$
g^{2}=\frac{8 G_{F} M_{W}^{2}}{\sqrt{2}} \quad \text { or } \quad g^{2} / \alpha=4 \pi \sin ^{2} \theta_{W},
$$

with $\alpha \approx 1 / 128$. The vector and axial neutral couplings are

$$
g_{V}^{i} \equiv t_{3 L}(i)-2 q_{i} \sin ^{2} \theta_{W}, \quad g_{A}^{i} \equiv t_{3 L}(i),
$$

where $t_{3 L}(i)$ is the weak isospin of the fermion $i$ and $q_{i}$ is the charge of $\psi_{i}$ in units of $e$. 


\section{A. $e^{-} e^{-}$fixed target experiments}

With the spinors in Eqs. (4) we have obtained all the helicity amplitudes of Ref. [3] when $m_{e} \rightarrow 0$; even with the actual value of $m_{e}$ the numerical value for the $A_{R L}(e e)$ asymmetry coincides with that obtained in Ref. [3] (i.e., at the tree level) as it should be. In fact, neglecting the lepton masses we obtain the asymmetry for a FT experiment in the ESM context:

$A_{R L}^{\mathrm{FT}, \mathrm{ESM}}(l l \rightarrow l l) \approx-\frac{G_{F} Q^{2}}{\sqrt{2} \pi \alpha} \frac{1-y}{1+y^{4}+(1-y)^{4}}\left(1-4 \sin ^{2} \theta_{W}\right)$,

where $Q^{2}=-\left(p_{1}^{\prime}-p_{1}\right)^{2}, \quad y=\sin ^{2}(\theta / 2)$, and the other constants are the well knowing $\alpha, G_{F}$, and $\sin ^{2} \theta_{W}$. For FT experiments we have $Q^{2}=-q^{2}=y\left(p_{1}+p_{1}^{\prime}\right)$ $=y\left(2 m_{l_{1}}^{2}+2 m_{l_{1}} E_{\text {beam }}\right)$. In Eq. (15) the approximation $m_{e}^{2}$ $\ll Q^{2} \ll M_{Z}^{2}$ was used. Also terms of order $m_{l} / E_{\text {beam }}$ were neglected. This approximation is valid even for $E_{\text {beam }} \approx 1$ $\mathrm{TeV}$ (typical energies of the NLC) since in this case $Q^{2}$ $\approx 0.5(\mathrm{GeV})^{2}$.

We see that for a FT experiment, the $A_{R L}$ asymmetry is small; in addition to the factor $1-4 \sin ^{2} \theta_{W}$ we have a small $Q^{2}$ [7]. Using the helicity amplitudes given in Appendixes A and B, with $E_{\text {beam }}=50 \mathrm{GeV}, \quad \theta \approx 90^{\circ} \quad(y=1 / 2)$, and for $100 \%$ beam polarization, we obtain

$$
A_{R L}^{\mathrm{FT}, \mathrm{ESM}}(e e \rightarrow e e) \approx-3 \times 10^{-7} .
$$

Hence, for FT experiments we have confirmed the value obtained in Ref. [3] for the case of $e^{-} e^{-}$at the tree level:

$$
A_{R L}^{\mathrm{FT}, \mathrm{ESM}}(e e \rightarrow e e) \approx-6 \times 10^{-9}\left(E_{\mathrm{beam}} / 1 \mathrm{GeV}\right) .
$$

Radiative corrections reduce the tree level value for the $A_{R L}^{\mathrm{FT}, \mathrm{ESM}}$ asymmetry in $e^{-} e^{-}$by $40 \pm 3 \%$ [7]. Although the asymmetry is small in FT experiments, the cross section of the Møller scattering is high: $\sigma_{T} \approx 10^{3} \mathrm{nb}$. In Eq. (C1) we show the differential cross section for the fixed target case.

The $A_{R ; R L}^{\mathrm{FT}, \mathrm{ESM}}$ asymmetry defined in Eq. (10) is shown in Fig. 1 as a function of $\sin ^{2}(\theta / 2)$. This asymmetry is dominated in the context of the ESM by purely QED effects $[8,9]$. However, we will see that it sensible for purely electroweak effects. In fact, for massless electrons we have (see Table I)

$$
\begin{aligned}
A_{R ; R L}^{\mathrm{FT}, \mathrm{ESM}}(e e \rightarrow e e) & \\
= & \frac{1-y^{4}-(1-y)^{4}}{y^{2}(1-y)^{2}}+s \beta_{W}\left(g_{V}^{2}+g_{A}^{2}+4 g_{V} g_{A}\right)+O\left(\beta_{W}^{2}\right) \\
\frac{1+y^{4}+(1-y)^{4}}{y^{2}(1-y)^{2}}+s \beta_{W}\left(3 g_{V}^{2}+g_{A}^{2}+4 g_{V} g_{A}\right)+O\left(\beta_{W}^{2}\right) &
\end{aligned}
$$

where $\beta_{W}=g^{2} / 4 \pi \alpha M_{Z}^{2} \approx 5.2 \times 10^{-4}$. Notice, however, that there are electroweak contributions which do not have the suppression factor $1-4 s_{W}^{2}$. Hence, the last effects are greater than in the case when the target is unpolarized, i.e.,

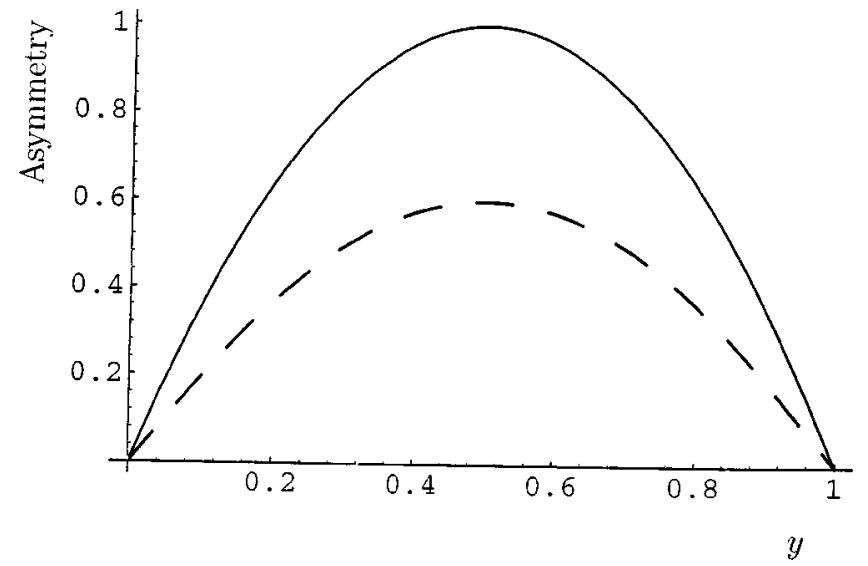

FIG. 1. The asymmetry with both beams polarized defined in Eq. (10) in a fixed target experiment for the process $e^{-} e^{-}$ $\rightarrow e^{-} e^{-}$as a function of $y=\sin ^{2}(\theta / 2)$, where $\theta$ is the center of mass scattering angle (solid line) and the same quantity but only considering the photon contributions given in Appendix A 1 (dashed line).

for the case of the asymmetry defined in Eq. (7). This is due to the fact that the contributions proportional to $g_{A}^{2}$ do not cancel out. When we calculate the asymmetry with the target unpolarized the cancellation occurs and we obtain the expression given in Eq. (15). In Fig. 1 the $A_{R ; R L}$ asymmetry appears when all amplitudes in Appendix A 1 are considered. In Fig. 1 we also show the same asymmetry but only considering the photon amplitudes which are given in Appendix A 1 . We note that, in fact, electroweak contributions are important as can be seen by comparing Figs. 1 and 2 .

For theoretical purely QED calculations in the Born approximation see Refs. $[8,10]$ and for radiative correction see Ref. [10]. For experimental data see Ref. [9]. The difference with the theoretical calculations $[8,10]$ is due to the electroweak effects that we have considered. This indicates that this asymmetry is sensitive to massive vector boson exchanges. Measurements of the QED contribution to the $A_{R ; R L}$ asymmetry (i.e., with both the incident beam and target longitudinally polarized) in a fixed target experiment with a beam energy up to near $20 \mathrm{GeV}$ are given in Ref. [9].

\section{B. $e^{-} e^{-}$collider experiments}

Next, let us consider a collider experiment with the interactions given in Eq. (12). In this case the transferred momentum is

$$
\begin{aligned}
Q^{2}= & -\left(p_{1}^{\prime}-p_{1}\right)^{2} \\
= & -m_{l_{1}^{\prime}}^{2}-m_{l_{1}}^{2}+\frac{s}{2} \\
& -2 \cos \theta\left[\left(\frac{s}{4}-m_{l_{1}}^{2}-m_{l_{1}^{\prime}}^{2}\right)\left(\frac{s}{4}-2 m_{l_{1}}^{2}\right)\right]^{1 / 2} \\
\rightarrow & s \sin ^{2} \frac{\theta}{2}, \quad \text { when } \quad m_{l_{i}} \rightarrow 0 .
\end{aligned}
$$

Hence, now $Q^{2}$ is not a small factor. 
TABLE I. Nonvanishing contributions in the ESM when the lepton mass is zero. Here $\beta_{W}$ $=g^{2} / 4 \pi \alpha M_{Z}^{2}$.

\begin{tabular}{lcclc}
\hline \hline InitiallFinal & $R R$ & $R L$ & $L L$ & $L R$ \\
\hline$R R$ & $\frac{1}{y(y-1)}+2 s \beta_{W}\left(g_{V}+g_{A}\right)^{2}$ & 0 & 0 & 0 \\
$R L$ & 0 & $\frac{y}{1-y}+s y \beta_{W}\left(g_{V}^{2}-g_{A}^{2}\right)$ & 0 & $\frac{1-y}{y}+s \beta_{W}(1-y)\left(g_{V}^{2}-g_{A}^{2}\right)$ \\
$L R$ & 0 & $\frac{1-y}{y}+s \beta_{W}(1-y)\left(g_{V}^{2}-g_{A}^{2}\right)$ & 0 & $\frac{y}{1-y}+s y \beta_{W}\left(g_{V}^{2}-g_{A}^{2}\right)$ \\
$L L$ & $\frac{y}{1-y}+2 s \beta_{W}\left(g_{V}+g_{A}\right)^{2}$ & 0 & 0 & 0 \\
\hline \hline
\end{tabular}

As we said before, in the ESM the total cross section is $\approx 1000 \mathrm{nb}$ in FT experiments and $\approx 10^{-3} \mathrm{nb}$ in collider experiments at $\sqrt{s}=300 \mathrm{GeV}$. [See Eqs. (C1) and (C2) for the differential cross section in the context of the electroweak standard model for collider and fixed target experiments, respectively.] So the required luminosity, or the parameter to be measured, in colliders must be larger than in fixed target experiments. According to Czarnecki and Marciano [7] the number of scattering events required for a $10^{-8}$ statistical accuracy in FT experiments is of the order of $10^{16}$. However, since the cross section for Møller scattering in FT experiments is large at low $Q^{2}$, the above requirement does not intimidate us. On the other hand, in collider experiments the cross section is rather smaller than that of FT experiments at high energies. Notwithstanding, as in this case the asymmetry is $\sim 10^{5}$ times the corresponding value for FT experiments [11] [see Eq. (20) below], the $10^{4}$ events/yr, which is the number of events expected for a luminosity of $\mathcal{L} \approx 10^{33} \mathrm{~cm}^{-2} \mathrm{~s}^{-1}$, will provide statistics just enough $\left(\sqrt{N} / N \sim 10^{-2}\right)$ to measure, although poorly measure, the $A_{R L}$ asymmetry in collider experiments. In addition, if new physics does really exist at the hundreds of $\mathrm{GeV}$ level, the cross section in colliders can serve to detect new resonances. If this is indeed the case, the cross section will have typical resonance enhancement(s). Recall also that the collider measurements of $A_{R L}$ in Møller scattering unlike the case of FT experiments will not have a background from $e N$ scattering.

Thus, it is worth considering the contribution to the $A_{R L}$ asymmetry at the energies of next linear colliders (electrons up to $1.5 \mathrm{TeV}$ ) and also in muon colliders (muons up to 4 $\mathrm{TeV}$ ). Although a large polarization implies sacrifice in luminosity at a muon collider [2], new physics (if any) with a clear signature could be distinguished even with low luminosity. Anyway, the asymmetry depends more on the polarization than on the luminosity.

At energies $\sqrt{s}=300 \mathrm{GeV}$ and $\theta \approx 90^{\circ}$ (in the center of mass frame) the asymmetry is

$$
A_{R L}^{\mathrm{CO}, \mathrm{ESM}}(e e \rightarrow e e) \approx-0.05 .
$$

Compare with the value for the FT experiment given in Eq. (16). In Fig. 2 we show the $A_{R L}^{\text {CO,ESM }}$ asymmetry as a function of $\sqrt{s}$ and $\theta$. Notice that this asymmetry is a smooth function of both variables.

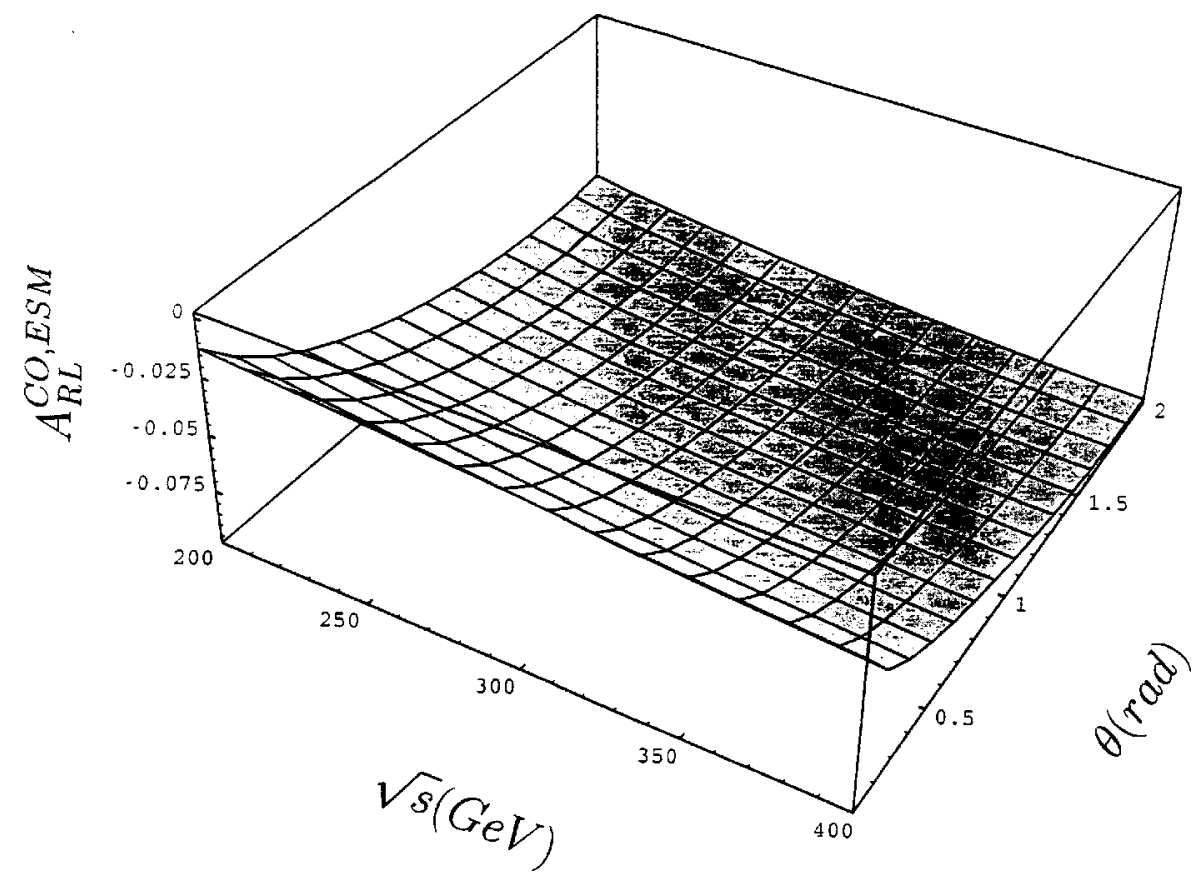

FIG. 2. The asymmetry $A_{R L}^{\mathrm{CO} \text { ESM }}$ defined in Eq. (7) for the $e^{-} e^{-} \rightarrow e^{-} e^{-}$reaction as a function of $\sqrt{s}$ and the angle $\theta$. 


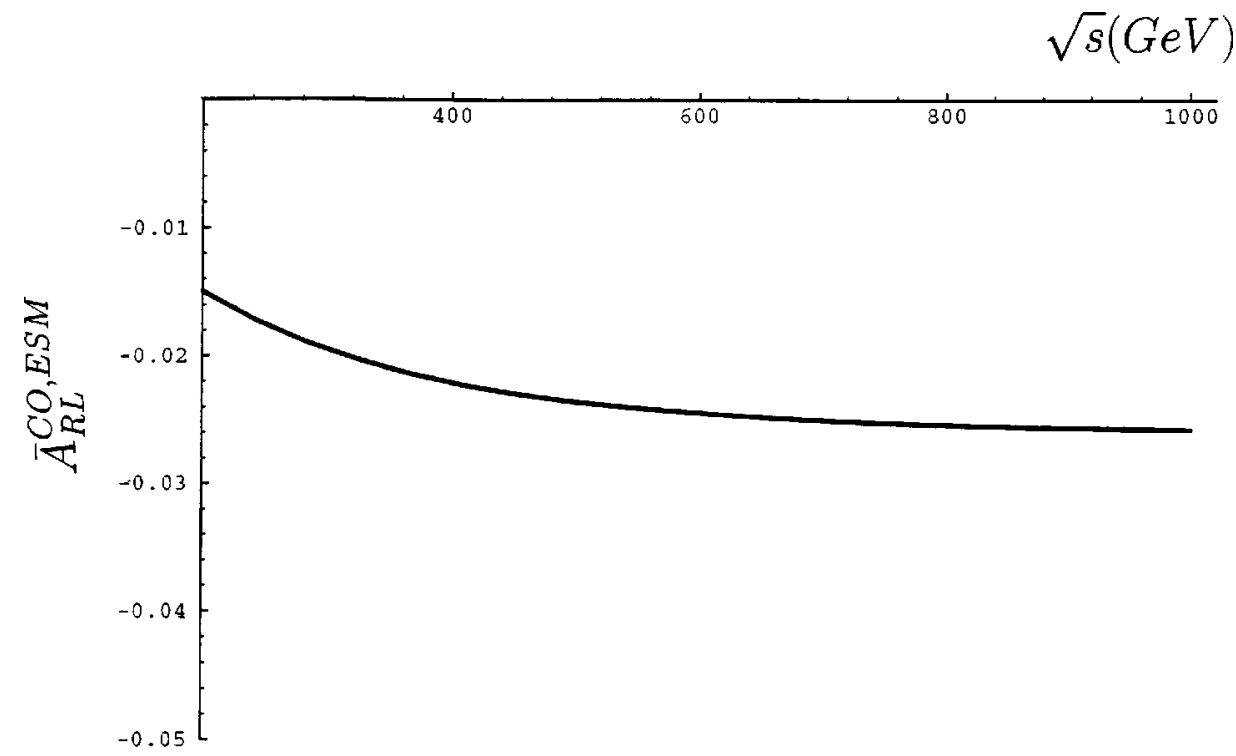

FIG. 3. The angular-integrated asymmetry $\bar{A}_{R L}^{\mathrm{CO}, \mathrm{ESM}}$ defined in Eq. (21) for the $e^{-} e^{-} \rightarrow e^{-} e^{-}$reaction as function of $\sqrt{s}$.

We can integrate in the scattering angle and define the asymmetry $\bar{A}_{R L}$ as

$$
\begin{aligned}
\bar{A}_{R L}(l l & \rightarrow l l) \\
= & \frac{\left(\int d \sigma_{R R}+\int d \sigma_{R L}\right)-\left(\int d \sigma_{L L}+\int d \sigma_{L R}\right)}{\left(\int d \sigma_{R R}+\int d \sigma_{R L}\right)+\left(\int d \sigma_{L L}+\int d \sigma_{L R}\right)},
\end{aligned}
$$

where $\int d \sigma_{i j} \equiv \int_{5^{\circ}}^{175^{\circ}} d \sigma_{i j}$. This integrated asymmetry for $e e \rightarrow e e, \quad$ in the electroweak standard model $\left[\bar{A}_{R L}^{\mathrm{CO}, \mathrm{ESM}}(l l \rightarrow l l)\right]$, appears in Fig. 3 as a function of $\sqrt{s}$. We see that this integrated asymmetry varies slowly with $\sqrt{s}$ and it is almost constant at the value -0.02 above $\sqrt{s}$ $=400 \mathrm{GeV}$.

\section{C. $\mu^{-} \mu^{-}$experiments}

A hypothetical fixed target $\mu^{-} \mu^{-}$experiment at the same $E_{\text {beam }}$ energy used in Sec. II A gives $A_{R L}(\mu \mu) \approx 5.4 \times 10^{-5}$ for $\theta \approx 90^{\circ}$. In this case $Q_{\text {muon }}^{2}=\left(m_{e} / m_{\mu}\right) Q_{\text {electron }}^{2}$ $\approx 4.2 \mathrm{GeV}^{2}$. A more realistic case is the $\mu^{-} \mu^{-}$collider where, for the same experimental conditions as in the previous subsection, we obtain

$$
A_{L R}^{\mathrm{CO}, \mathrm{ESM}}(\mu \mu \rightarrow \mu \mu) \approx-0.1436 .
$$

Notice the large value for the asymmetry for the muons with respect to the electron case given in Eq. (20).

In the electron case the Higgs contributions are negligible. For the muon case, if we do not take into account the Higgs amplitudes given in Appendix A 1, the asymmetry in Eq. (22) has the value of -0.1437 , showing a slight dependence on the Higgs contributions. There are also effects of the lepton mass in the $\gamma, Z$ contributions to the asymmetry that are responsible for the different values in Eqs. (20) and (22). Independently of the numerical value of the Higgs contributions, the important issue is that all its main contributions (see the amplitudes given in Sec. A 3) cancel out in the $A_{R L}$ asymmetry defined in Eq. (7). In fact, as we will see in the next section, even in models with larger scalar-lepton couplings the extra scalar contributions cancel out.

\section{LEFT-RIGHT ASYMMETRIES IN BILEPTON MODELS}

An example of possible new physics are models with a doubly charged vector boson [12]. Then, the charged current interaction in terms of the physical basis is given by

$$
\begin{gathered}
-\frac{g}{\sqrt{2}}\left[\bar{\nu}_{L} E_{L}^{\nu^{\dagger}} E_{L}^{l} l_{L} W_{\mu}^{+}+\bar{l}_{L}^{c} \gamma^{\mu} E_{R}^{l T} E_{L}^{\nu} \nu_{L} V_{\mu}^{+}\right. \\
\left.-\bar{l}_{L}^{c} E_{R}^{l T} E_{L}^{l} l_{L} U_{\mu}^{++}\right]+ \text {H.c. },
\end{gathered}
$$

with $\quad l_{L}^{\prime}=E_{L}^{l} l_{L}, \quad l_{R}^{\prime}=E_{R}^{l} l_{R}, \quad \nu_{L}^{\prime}=E_{L}^{\nu} \nu_{L}, \quad$ the primed (unprimed) fields denoting symmetry (mass) eigenstates. We see from Eq. (23) that for massless neutrinos we have no mixing in the charged current coupled to $W_{\mu}^{+}$but we still have mixing in the charged currents coupled to $V_{\mu}^{+}$and $U_{\mu}^{++}$. That is, if neutrinos are massless, we can always choose $E_{L}^{\nu \dagger} E_{L}^{l}=1$. However, the charged currents coupled to $V_{\mu}^{+}$and $U_{\mu}^{++}$are not diagonal in flavor space and the mixing matrix $\mathcal{K}=E_{R}^{l T} E_{L}^{\nu}$ has three angles and three phases. (An arbitrary $3 \times 3$ unitary matrix has three angles and six phases. In the present case, however, the matrix $\mathcal{K}$ is determined entirely by the charged lepton sector; so we can rotate only three phases [13].)

If the bilepton $U_{\mu}^{++}$is not too heavy, say, with a mass of the order of a few hundred $\mathrm{GeV}$, it is possible that it will appear as a resonance in the next linear colliders. Assuming that the matrix $\mathcal{K}$ is almost diagonal we can neglect the mixing effects in processes involving the same initial and final 
leptons as $l^{-} l^{-} \rightarrow l^{\prime-} l^{\prime-}$. The helicity amplitudes for $U$ exchange in the $s$ channel are given, at the tree level, in Appendix D. We do not know yet the total width $\Gamma_{U}$ of the $U^{--}$bilepton; however, we know that at least it has to decay into $l^{-} l^{\prime-}$ : If these were the only channels, we have, for the width,

$$
\Gamma\left(U^{--} \rightarrow l_{i}^{-} l_{j}^{-}\right)=\frac{G_{F} M_{U}^{3}}{6 \sqrt{2} \pi}\left|\mathcal{K}_{i j}\right|^{2},
$$

and if $\mathcal{K}_{i j}$ is almost diagonal, the main decays will be $U$ $\rightarrow e^{-} e^{-}, \mu^{-} \mu^{-}, \tau^{-} \tau^{-}$; with an illustrative value of $M_{U}$ $=300 \mathrm{GeV}$, we have $\Gamma(U \rightarrow$ leptons $) \approx 36 \mathrm{GeV}$. Recent analyses based on the cross section for the reaction $e^{-} e^{-}$ $\rightarrow \mu^{-} \mu^{-}$using effective interactions consider doubly charged vector bileptons of mass $200 \mathrm{GeV}$ for an effective constant coupling of the order of 0.1 [14] which corresponds to our $\mathcal{K}_{i j} \approx 1$ case. In models with such a vector bilepton there are several Higgs fields and also exotic quarks. Hence, we should have to take into account $U^{--} \rightarrow$ scalars and also $U^{--} \rightarrow d_{3} \bar{J}^{-5 / 3}, \bar{u}_{1}\left(\bar{u}_{2}\right) J^{-4 / 3}$, with $d_{3}$ and $u_{1,2}$ denoting symmetry eigenstates of the known quarks and $J^{Q}\left(\bar{J}^{Q}\right)$ denotes an exotic quark (antiquark) of electric charge $Q$. The lefthanded mixing matrices, defined by the relation among the symmetry eigenstates (primed fields) with mass eigenstates (unprimed fields) i.e., $U_{L}^{\prime}=V_{L}^{u} U_{L}$ and $D_{L}^{\prime}=V_{L}^{d} D_{L}$, survive in the Lagrangian density. Thus, the partial width is of the form (neglecting the masses of the quarks $J$ )

$$
\Gamma\left(U^{--} \rightarrow d_{3} \bar{J}^{-5 / 3}\right)=\frac{C G_{F} M_{U}^{3}}{6 \pi \sqrt{2}}\left|\left(V_{L}^{d}\right)_{3 i}\right|^{2},
$$

and similarly for the other decays into $u$ quarks. Notice that the mixing matrix in Eq. (25) is not necessarily equal to the Cabibbo-Kobayashi-Maskawa mixing matrix which is defined as $V_{\mathrm{CKM}}=V_{L}^{u^{\dagger}} V_{L}^{d}$. The parameters in both Eqs. (24) and (25) are constrained for other processes but here we will not consider these constraints. A realistic calculation will have to take into account the masses of the exotic quarks $J$. At present, however, there are no experimental data bounds on the values of these masses. Of course, there are also trilinear vector interactions $U^{--} \rightarrow W^{-} V^{-}$that it will occur if $M_{V}+M_{W}<M_{U}$ ( $V^{-}$is a singly charged bilepton present in the model). As a detailed calculation of $\Gamma_{U}$ is out of the scope of this work, we will consider $\Gamma_{U}$ as a free parameter.

In this model the Yukawa couplings between leptons and a sextet of scalars transforming as $(6,0)$ under $\mathrm{SU}(3)_{L}$ $\otimes \mathrm{U}(1)_{N}$ are of the form $g_{a b}\left(\overline{\left.\psi_{a i L}\right)^{c}} \psi_{b j L} S_{i j}\right.$ where $a, b$ $=e, \mu, \tau ; \quad i, j$ are $\mathrm{SU}(3)$ indices and the matrix $g_{a b}$ denotes a symmetrical matrix. If this is the only lepton-Yukawa interaction in the model, by using a discrete symmetry we can avoid the coupling to the triplet $\eta \sim(3,0)$; there is no flavorchanging neutral current (FCNC) in this sector and the lepton-Higgs sextet couplings are proportional to $m_{l} / v_{S}$ where $m_{l}$ is the lepton mass and $v_{S}$ is the vacuum expectation value (VEV) of the neutral component of the sextet. Since there are already two other scalar triplets which give the appropriate mass to the $W^{ \pm}$and $Z^{0}$ vector bosons, it is not necessary that $v_{S}$ be of the same order of magnitude than the other vacuum expectation values that are present in the model. Hence, $v_{S}$ may be of the order of a few $\mathrm{GeV}$ since the heavier lepton is the $\tau$ with a mass of $1.777 \mathrm{GeV}$ [15]. Notice that since the neutral part of the sextet is a doublet of SU(2), these fields have $\rho \equiv M_{W}^{2} / M_{Z}^{2} \cos ^{2} \theta_{W}=1$ at the tree level. The doubly charged member $H_{1}^{++}$is part of a triplet with its neutral partner having vanishing vacuum expectation value (if neutrinos do not get Majorana masses). There is also a doubly charged $\mathrm{H}_{2}^{++}$which is a singlet of $\mathrm{SU}(2)$. Strictly speaking the VEV which is in control of $\rho$ (and flavor-changing neutral currents coupled to $Z$ ) is that of the triplet which breaks the $\mathrm{SU}(3)_{L} \otimes \mathrm{U}(1)_{N}$ symmetry. For an arbitrary high value for this VEV we can obtain the ESM predictions. At the one-loop level it is possible that a finetuning is required but this deserve a more detailed study.

From the experimental point of view a lower bound on the mass of doubly charged scalars is $m_{H^{+}+}>45 \mathrm{GeV}[16,17]$ but we recall that the phenomenology of the $\mathrm{H}^{++}$derives from its couplings. The processes which have to be considered are $\gamma / Z \rightarrow H^{++} H^{--}$and $H^{--} \rightarrow l^{-} l^{\prime-}$ [18]. In the model of Ref. [12] the coupling $W^{-} W^{-}$with doubly charged scalars does not exist (the vertex always includes a single charged vector bilepton $V^{-}$; i.e., the coupling which really exists is with $V^{-} W^{-}$).

Although the bilepton model we consider here is a gaugeinvariant theory which has its own scalar spectrum, we will consider in this work the bilepton contributions as extra contributions to the electroweak standard model ones due to the fact that in any case the neutral Higgs contributions are smaller than that of the doubly charged vector bilepton. We denote this by using the $\{E S M\}$ and $\{E S M+U\}$ labels in cross sections and asymmetries. However, as we will show, the doubly charged scalar bilepton contributions, denoted by $\{\mathrm{ESM}+\mathrm{H}\}$, cancel out in the numerator of the $A_{R L}$ asymmetry. Hence, in practice our calculations can be considered a true effect of the bilepton model of Ref. [12].

\section{A. $e^{-} e^{-}$high energy colliders}

By using the expression in Eqs. (C1) and (D6) we can compute the effect of such bilepton fields. In Fig. 4 we show

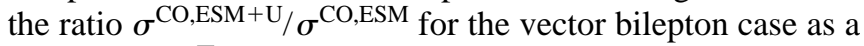
function of $\sqrt{s}$ and $\Gamma_{U}$. We observe that this ratio becomes appreciable for $\sqrt{s}$ near the $U$ resonance as expected. In this case we have chosen an arbitrary (but reasonable) value of $M_{U}=300 \mathrm{GeV}$ to illustrate the cross section behavior. We also note that this quantity is smoothly dependent on the $\Gamma_{U}$ values. Since the cross section could be large at the $U$ peak, if the mass of the $U$ is lower than the energies of future collider experiments, it is interesting to know the value of the $A_{R L}$ asymmetry at these energies.

Here, we will not show the analytic expression of $A_{R L}^{\mathrm{CO}}$, but at the same conditions as Eq. (20) it gives

$$
A_{R L}^{\mathrm{CO}, \mathrm{ESM}+\mathrm{U}}(e e \rightarrow e e)=-0.099,
$$




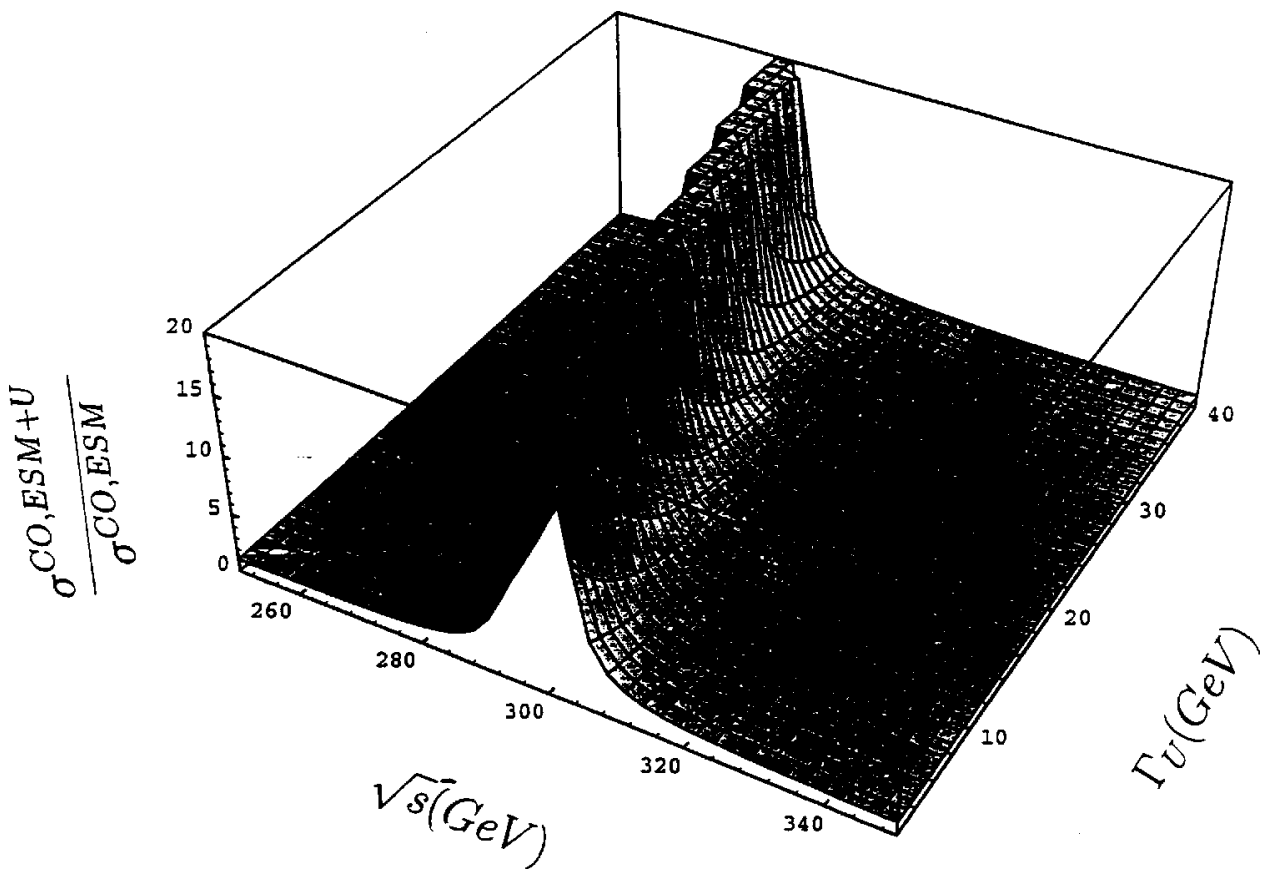

FIG. 4. The ratio $\sigma^{\mathrm{CO}, \mathrm{ESM}+\mathrm{U}} / \sigma^{\mathrm{CO}, \mathrm{ESM}}$ for the total cross section obtained from Eq. (D6) which includes the $s$-channel $U^{--}$contribution and Eq. (C2) for the ESM for the reaction $e^{-} e^{-} \rightarrow e^{-} e^{-}$as a function of $\sqrt{s}$ and $\Gamma_{U}$.

and we see that it is larger than the respective value obtained in the context of the ESM [see Eq. (20)]. A more illustrative quantity is the angular-integrated asymmetry defined in Eq. (21) and denoted here by ${\overline{A_{R L}}}^{\mathrm{CO}, \mathrm{ESM}+\mathrm{U}}(e e \rightarrow e e)$. We show it in Fig. 5 as a function of $\sqrt{s}$ and $\Gamma_{U}$. We can observe that the dependence of $\Gamma_{U}$ cancels out in $\overline{A_{R L}} \mathrm{CO}, \mathrm{ESM}+\mathrm{U}(e e$ $\rightarrow e e$ ), so that even if we do not use a realistic calculation for $\Gamma_{U}$ based in all possible decay channels predicted by the model, the result will be, in fact, independent of it. As expected the asymmetry is larger in the $U$ pole. In Fig. 6 we quote the quantity

$$
\delta \bar{A}_{R L}(e e \rightarrow e e) \equiv\left(\bar{A}_{R L}^{\mathrm{CO}, \mathrm{ESM}+\mathrm{U}}-\bar{A}_{R L}^{\mathrm{CO}, \mathrm{ESM}}\right) / \bar{A}_{R L}^{\mathrm{CO}, \mathrm{ESM}}
$$

with the $\bar{A}$ 's defined in Eq. (21), as a function of $\sqrt{s}$ and $\Gamma_{U}$.

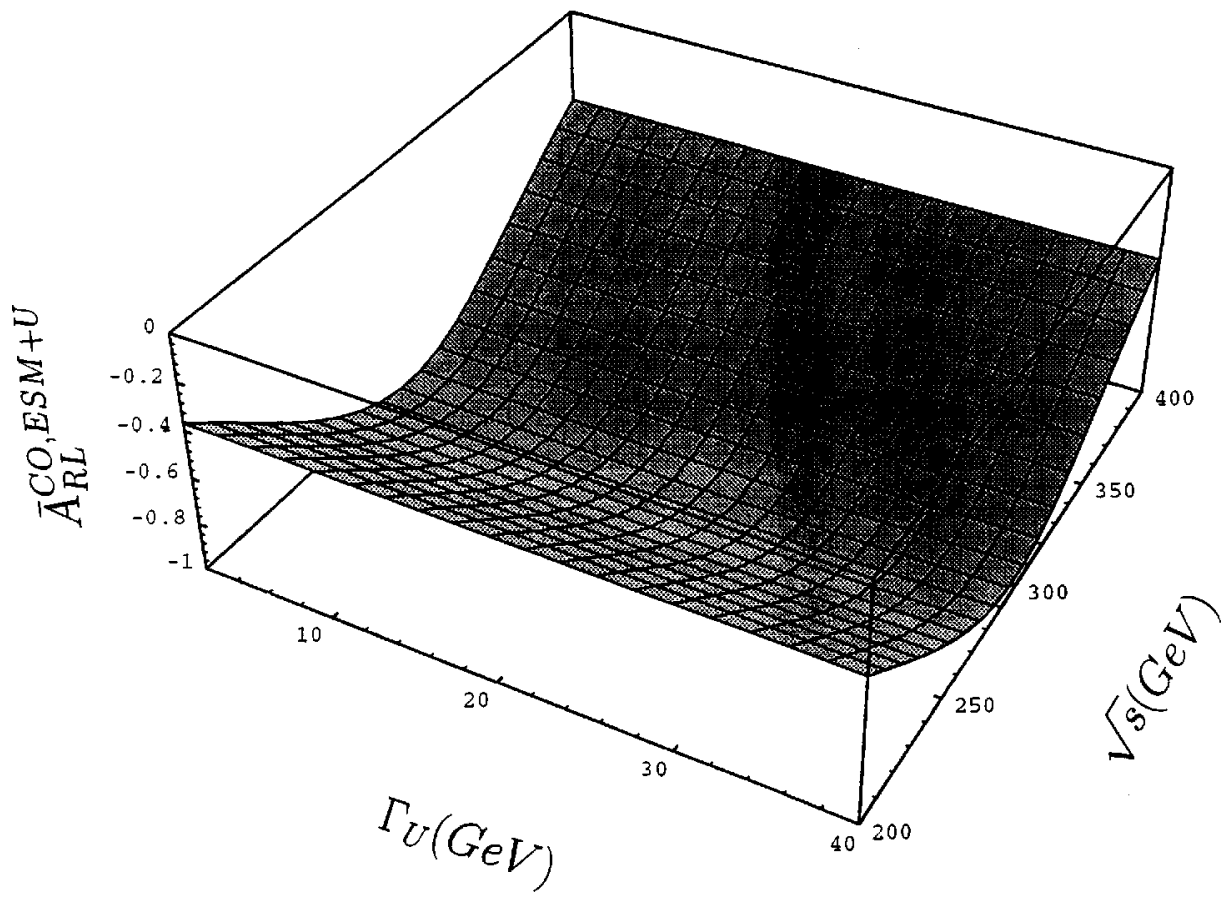

FIG. 5. Same as Fig. 4 but taking into account the contributions of the bilepton $U^{--}$as a function of $\sqrt{s}$ and $\Gamma_{U}$. 


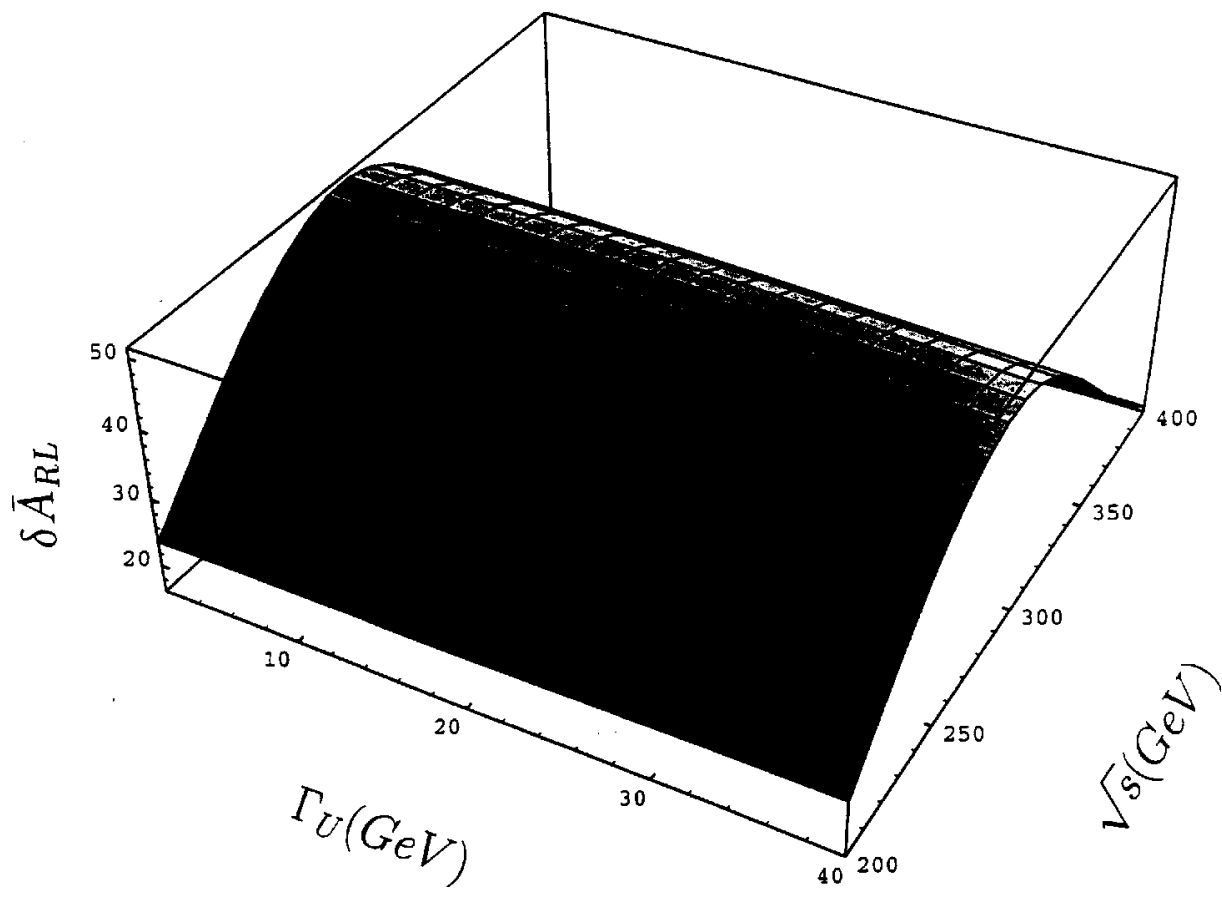

FIG. 6. $\delta \overline{A_{R L}}$ defined in Eq. (27) as a function of $\Gamma_{U}$ and $\sqrt{s}$ for $e^{-} e^{-} \rightarrow e^{-} e^{-}$.

As observed above this quantity is independent of $\Gamma_{U} \cdot \delta \overline{A_{R L}}$ is large at the $U$ resonance; however, we would like to stress that it remains appreciably large even far from the $U$ peak. That particular behavior suggests that this quantity could be the one to be considered in the search for new physics, like the bilepton $U^{--}$, in future colliders.

Next let us consider the scalar contribution in the model. The $s$-channel amplitudes appear in Appendix E. In this model the amplitudes in Appendix A 3 are used by making $v \rightarrow v_{S}$, and, as we said before, in models with several scalar multiplets it is possible that one of the neutral scalars does not contribute significantly to the $W^{-}$and $Z$ masses. It means that the VEV of such a scalar can be of the order of a few $\mathrm{GeV}$. In this case there are some scalar contributions which are proportional to $m_{l} / v_{S}$ with $m_{l}$ denoting the lepton mass and $v_{S}$ is a VEV which gives mass for the leptons.

Here we will use $M_{H_{i}}^{++}=50,300 \mathrm{GeV}$ (for both $i=1,2$ although the masses of the triplet and singlet might be different) and $v_{S}=10 \mathrm{GeV}$ only as an illustration. We also take for colliders $\sqrt{s}=300 \mathrm{GeV}$. Taking into account the ESM plus the scalar contribution (but not the vector $U^{--}$contributions) we obtain

$$
A_{R L}^{\mathrm{CO}, \mathrm{ESM}+\mathrm{H}}(e e \rightarrow e e)=-0.05,
$$

for $y=1 / 2$. We see that this value is the same as the pure ESM contribution given in Eq. (20). This is not a surprise since the purely scalar contributions cancel out in both the neutral Higgs (as in Appendix A 3) and also in the doubly charged Higgs contributions as can be seen from the amplitudes in Appendix E and the definition of the asymmetry in Eq. (7). However, in the muon-muon case the interference terms among the neutral Higgs and $\gamma, Z$ contributions are relatively significant as we will see in the next subsection.

\section{B. $\mu^{-} \mu^{-}$high energy colliders}

We can calculate all the above asymmetries in the case of a possible $\mu^{-} \mu^{-}$collider. For instance taking into account the contributions of the $U^{--}$vector bilepton we obtain

$$
A_{R L}^{\mathrm{CO}, \mathrm{ESM}+\mathrm{U}}(\mu \mu \rightarrow \mu \mu)=-0.1801,
$$

while the contribution of the $H^{--}$scalar bilepton gives

$$
A_{R L}^{\mathrm{CO}, \mathrm{ESM}+\mathrm{H}}(\mu \mu \rightarrow \mu \mu)=-0.1436,
$$

for $M_{H}^{++}=50$ and $300 \mathrm{GeV}$.

As discussed above, in the model with bileptons the VEV of the neutral scalar coupled mainly with leptons does not need to be equal to the VEV of the ESM ( $v \sim 246 \mathrm{GeV})$ but it can have a lower value, say, $v_{S} \sim 10 \mathrm{GeV}$. For the muon case we calculate the asymmetry in Eq. (29) with and without the ESM Higgs scalar contributions given in Appendix A 3. The respective values are -0.1436 and -0.1442 . If the mass of the $U^{--}$were $500 \mathrm{GeV}$, the asymmetry in Eq. (29) has the value -0.0133 .

\section{CONCLUSIONS}

We have shown in this work that collider experiments can be suitable for studying the left-right asymmetries in leptonlepton scattering. In fact, we have shown that in this case the asymmetries are larger than those which appear in fixed target experiments. In particular we have shown that there are significant differences between electron and muon asymmetries, the muon ones being larger than the electron ones due to mass effects. However, more details were given here for the electron case. For instance, the $A_{R L}$ asymmetry, when the contributions of the $U^{--}$vector bilepton are considered, is 
TABLE II. Nonvanishing contributions of the $U$-vector bosons when the lepton mass is zero.

\begin{tabular}{lcccc}
\hline \hline InitiallFinal & $R R$ & $R L$ & $L L$ & $L R$ \\
\hline$R R$ & 0 & 0 & 0 & 0 \\
$R L$ & 0 & $-2(1-y)$ & 0 & $2 y$ \\
$L R$ & 0 & $-2 y$ & 0 & $2(1-y)$ \\
$L L$ & 0 & 0 & 0 & 0 \\
\hline \hline
\end{tabular}

larger than the ESM value, as can be seen from Fig. 6 for the electron-electron case. On the other hand, the purely scalar bilepton contributions cancel out in the asymmetries as can be seen from the expression given in Appendixes A 3 and E. Only the interference terms between the scalars and $\gamma, Z$ amplitudes survive and these are not negligible in the muonmuon case.

Concerning the asymmetry $A_{R ; R L}$ defined in Eq. (10) i.e., when both beams are polarized, we see from Table II that it is given by

$$
A_{R ; R L}^{\mathrm{CO}, \mathrm{ESM}+\mathrm{U}}(e e \rightarrow e e) \approx-1+O\left(\beta_{W}\right),
$$

and comparing with Eq. (18) we see that the maximal value of $A_{R ; R L}^{\mathrm{CO} E S M+\mathrm{U}}(e e \rightarrow e e)$ and its dependence on $y$ are different in models with vector bileptons and the electroweak standard model (almost purely QED) case. At the $U$ resonance $d \sigma_{R L}$ $\gg d \sigma_{R R}$, and hence $A_{R ; R L}^{\mathrm{CO}, E S M}+U(e e \rightarrow e e) \approx-1$. In the ESM on the contrary we have $d \sigma_{R R} \gg d \sigma_{R L}$, and hence $A_{R ; R L}^{\mathrm{CO}, \mathrm{ESM}}(e e \rightarrow e e) \approx+1$. Similarly, $A_{L ; R L}^{\mathrm{CO}, \mathrm{ESM}+\mathrm{U}}(e e \rightarrow e e) \approx$ -1 and $A_{L ; R L}^{\mathrm{CO}, \mathrm{ESM}}(e e \rightarrow e e) \approx+1$. Although this asymmetry is large in both the ESM (see Fig. 1) and the bilepton model the sign of it is opposite and could be useful for discovering such a vector field.

Summarizing, we have calculated in this work several left-right asymmetries in the context of the ESM and in models with both vector and scalar bileptons.
Finally, a remark: in some models with vector bileptons such as $U^{--}$the decay $\mu^{-} \rightarrow e^{-} e^{-} e^{+}$(and similar ones) does occur. The branching ratio for this decay is $\sim 10^{-12}$ [15]. This bound strongly limits the $\mathcal{K}_{e \mu}$ couplings. The branching fraction for $\mu \rightarrow 3 e$ decay is

$$
B(\mu \rightarrow 3 e) \propto\left(\frac{\mathcal{K}_{e \mu} \mathcal{K}_{e e}}{M_{U}^{2}}\right)^{2} \frac{1}{G_{F}^{2}}
$$

For $M_{U}=300 \mathrm{GeV}$ and $\mathcal{K}_{e e} \approx 1$ the experimental value for the above branching ratio implies $\mathcal{K}_{e \mu} \sim 10^{-6}$. The doubly charged scalar will also contribute to these rare decays. For a comprehensive analysis of the constraints on bilepton masses and their coupling constants with leptons and for several types of bileptons see Ref. [19].

\section{ACKNOWLEDGMENTS}

This work was supported by Fundação de Amparo à Pesquisa do Estado de São Paulo (FAPESP), Conselho Nacional de Ciência e Tecnologia $(\mathrm{CNPq})$ and by Programa de Apoio a Núcleos de Excelência (PRONEX).

\section{APPENDIX A: INVARIANT AMPLITUDES IN THE ESM}

In this appendix we give the invariant amplitudes for the Møller scattering in the context of the $\mathrm{SU}(2)_{L} \otimes \mathrm{U}(1)_{Y}$ (ESM) model. Although the Higgs boson in context of the ESM gives contributions that are negligible for practical proposes, we include its contribution. In models with a rich scalar spectrum and/or with exotic heavy fermions the scalar contribution may become important. We omit a common factor of $\left(2 E_{l}\right)^{2}$ in all amplitudes since it cancels out in the asymmetries for diagonal processes such as $e^{-} e^{-} \rightarrow e^{-} e^{-}$.

\section{Photon amplitudes}

Up to a factor $i e^{2} / t$ the $t$-channel amplitudes are [recall that $y=\sin ^{2}(\theta / 2), 1-2 y=\cos \theta$, and $\left.2 y^{1 / 2}(1-2 y)=\sin \theta\right]$

$$
\begin{gathered}
M_{R R ; R R}^{\gamma}(t)=\frac{4 E_{l}^{2}+[(1-2 y)-3] m_{l}^{2}}{2 E_{l}^{2}}, \quad M_{R R ; L R}^{\gamma}(t)=M_{R R ; R L}^{\gamma}(t)=\frac{m_{l}}{2 E_{l}} 2 y^{1 / 2}(1-2 y), \\
M_{R R ; L L}^{\gamma}(t)=\frac{m_{l}^{2}}{E_{l}^{2}} y, \\
M_{L R ; R R}^{\gamma}(t)=M_{L R ; L L}^{\gamma}(t)=M_{R R ; L R}^{\gamma}(t), \quad M_{L R ; L R}^{\gamma}(t)=\frac{2 E_{l}^{2}-m_{l^{\prime}}^{2}}{E_{l}^{2}}(1-y), \quad M_{L R ; L R}^{\gamma}(t)=M_{R R ; L L}^{\gamma}(t), \\
M_{R L ; R R}^{\gamma}(t)=M_{R L ; L L}^{\gamma}(t)=-M_{R R ; L R}^{\gamma}(t), \quad M_{R L ; L R}^{\gamma}(t)=M_{L R ; L R}^{\gamma}(t), \quad M_{R L ; L L}^{\gamma}(t)=M_{R R ; L L}^{\gamma}(t), \\
M_{L L ; R R}^{\gamma}(t)=M_{R R ; L L}^{\gamma}(t), \quad-M_{L L ; L R}^{\gamma}(t)=M_{L L ; R L}^{\gamma}(t)=M_{R R ; L R}^{\gamma} ; \quad M_{L L ; L L}^{\gamma}(t)=M_{R R ; R R}^{\gamma}(t),
\end{gathered}
$$

and the photon $u$-channel amplitudes, up to a factor $i e^{2} / u$, are, 


$$
\begin{gathered}
M_{R R ; R R}^{\gamma}(u)=\frac{4 E_{l}^{2}-[3+(1-2 y)] m_{l}^{2}}{2 E_{l}^{2}}, \quad M_{R R ; L R}^{\gamma}(u)=-M_{R R ; R L}^{\gamma}(u)=M_{R R ; L R}^{\gamma}(t), \\
M_{R R ; L L}^{\gamma}=\frac{m_{l}^{2}}{E_{l}^{2}}(1-y), \\
-M_{L R ; R R}^{\gamma}(u)=M_{L R ; L L}^{\gamma}(u)=M_{R R ; L R}^{\gamma}(t), \quad M_{L R ; L R}^{\gamma}(u)=\frac{m_{l}^{2}}{E_{l}^{2}} y, \quad M_{L R ; R L}^{\gamma}(u)=\frac{2 E_{l}^{2}-m_{l}^{2}}{E_{l}^{2}} y, \\
M_{R L ; R R}^{\gamma}(u)=-M_{R L ; L L}^{\gamma}=M_{R R ; L R}^{\gamma}(t), \quad M_{R L ; L R}^{\gamma}(u)=M_{L R ; R L}^{\gamma}(u), \quad M_{R L ; R L}^{\gamma}(u)=M_{R R ; L L}^{\gamma}(u), \\
M_{L L ; R R}^{\gamma}(u)=M_{R R ; L L}^{\gamma}(u), \quad M_{L L ; L R}^{\gamma}=M_{L L ; R L}^{\gamma}(u)=M_{R R ; L R}^{\gamma}(t), \quad M_{L L ; L L}^{\gamma}(u)=M_{R R ; R R}^{\gamma}(u) .
\end{gathered}
$$

\section{2. $Z$ amplitudes}

Up to a factor $i g^{2} /\left(t-M_{Z}^{2}\right)$, the $t$-channel $Z$-exchange amplitudes are

$$
\begin{gathered}
M_{R R ; R R}^{Z}(t)=\left[\frac{\left.4 E_{l}^{2}+[-3+(1-2 y)] m_{l}^{2}\right]}{2 E_{l}^{2}}\right]\left(g_{V}^{2}+g_{A}^{2}\right)-4\left(\frac{E_{l}^{2}-m_{l}^{2}}{E_{l}^{2}}\right)^{1 / 2} g_{V} g_{A}, \\
M_{R R ; L R}^{Z}(t)=\frac{m_{l}}{2 E_{l}}\left(g_{V}^{2}+g_{A}^{2}\right) 2 y^{1 / 2}(1-2 y), \quad M_{R R ; R L}^{Z}(t)=\frac{m_{l}}{2 E_{l}}\left(g_{V}^{2}-g_{A}^{2}\right) 2 y^{1 / 2}(1-2 y), \\
M_{R R ; L L}^{Z}(t)=\frac{m_{l}^{2}}{2 E_{l}^{2}}\left\{g_{V}^{2} y+g_{A}^{2}[3+(1-2 y)]\right\}, \\
M_{L R ; R R}^{Z}(t)=M_{L R ; L L}^{Z}(t)=M_{R R ; R L}^{Z}(t), \\
-M_{R L ; R R}^{Z}(t)=M_{R L ; L L}^{Z}(t)=M_{R R ; R L}^{Z}(t), \quad M_{R L ; L R}^{Z}(t)=M_{L R ; R L}^{Z}(t), \quad M_{R L ; R L}^{Z}=M_{L R ; L R}^{Z}(t), \\
M_{L L ; R R}^{Z}(t)=M_{R R ; L L}^{Z}(t), \quad M_{L L ; L R}^{Z}(t)=M_{L L, R L}^{Z}(t)=M_{R R ; L R}^{Z}(t), \quad M_{L L ; L L}^{Z}=M_{R R ; R R}^{Z}(t) .
\end{gathered}
$$

Up to a factor $i g^{2} /\left(u-M_{Z}^{2}\right)$, the $u$-channel $Z$-exchange amplitudes are

$$
\begin{gathered}
M_{R R ; R R}^{Z}(u)\left[\frac{\left.4 E_{l}^{2}-[3+(1-2 y)] m_{l}^{2}\right]}{E_{l}^{2}}\right]\left(g_{V}^{2}+g_{A}^{2}\right)-4\left(\frac{E_{l}^{2}-m_{l}^{2}}{E_{l}^{2}}\right)^{1 / 2} g_{V} g_{A}, \\
M_{R R ; L R}^{Z}(u)=M_{R R ; L R}^{Z}(t), \quad M_{R R ; R L}^{Z}(u)=-M_{R R ; R L}^{Z}(t), \\
M_{R R ; L L}^{Z}(u)=\frac{m_{l}^{2}}{2 E_{l}^{2}}\left\{g_{V}^{2}(1-y)+g_{A}^{2}[(1-2 y)-3]\right\}, \\
M_{L R ; R R}^{Z}(u)=M_{L R ; L L}^{Z}(u)=M_{R R ; R L}^{Z}(t), \quad M_{L R ; L R}^{Z}(u)=\frac{m_{l}^{2}}{E_{l}^{2}}\left(g_{V}^{2}-g_{A}^{2}\right)(1-y), \\
M_{L R ; R L}^{Z}(u)=\left(\frac{2 E_{l}^{2}-m_{l}^{2}}{E_{l}^{2}}\right)\left(g_{V}^{2}-g_{A}^{2}\right) y, \\
M_{R L ; R R}^{Z}(u)=M_{R L ; L L}^{Z}(u)=M_{R R ; R L}^{Z}(t), \quad M_{R L ; L R}^{Z}(u)=M_{L R ; R L}^{Z}(u), \quad M_{R L ; R L}^{Z}(u)=M_{L R ; L R}^{Z}(u),
\end{gathered}
$$




$$
\begin{gathered}
M_{L L ; R R}^{Z}(u)=M_{R R ; L L}^{Z}(u), \quad M_{L L ; L R}^{Z}(u)=M_{L L ; R L}^{Z}(u)=M_{R R ; R L}^{Z}(t), \\
M_{L L ; L L}^{Z}(u)=\left[\frac{4 E_{l}^{2}-[3+(1-2 y)] m_{l}^{2}}{E_{l}^{2}}\right]\left(g_{V}^{2}+g_{A}^{2}\right)+4\left(\frac{E_{l}^{2}-m_{l}^{2}}{E_{l}^{2}}\right)^{1 / 2} g_{V} g_{A} \cdot
\end{gathered}
$$

\section{3. $H^{0}$ amplitudes}

Here $H^{0}$ denotes a neutral Higgs boson with couplings like the ESM Higgs boson. Up to a common $i m_{l}^{2} / v^{2}\left(t-M_{H}^{2}\right)$ factor, the $t$-channel $H$-exchange amplitudes are

$$
\begin{gathered}
M_{R R ; R R}^{H}(t)=-\frac{m_{l}^{2}}{E_{l}^{2}}(1-y), \quad M_{R R ; L R}^{H}(t)=M_{R R ; R L}^{H}=-\frac{m_{l}}{2 E_{l}} 2 y^{1 / 2}(1-2 y), \quad M_{R R ; L L}^{H}(t)=y, \\
-M_{L R ; R R}^{H}(t)=M_{L R ; L L}^{H}(t)=M_{R R ; L R}^{H}(t) ; \quad M_{L R ; L R}^{H}(t)=M_{R R ; R R}^{H}(t), \quad M_{L R ; R L}^{H}(t)=M_{R R ; L L}^{H}(t), \\
-M_{R L ; R R}^{H}(t)=M_{R L ; L L}^{H}(t)=M_{R R ; L R}^{H}(t), \quad M_{R L ; L R}^{H}(t)=M_{R R ; L L}^{H}(t), \quad M_{L R ; R L}^{H}(t)=M_{R R ; R R}^{H}(t), \\
-M_{L L ; R R}^{H}(t)=M_{R R ; L L}^{H}(t) ; \quad-M_{L L ; L R}^{H}(t)=-M_{L L ; R L}^{H}(t)=M_{R R ; L R}^{H}(t), \quad M_{L L ; L L}^{H}(t)=M_{R R ; R R}^{H}(t) .
\end{gathered}
$$

Up to a common $i m_{l}^{2} / v^{2}\left(u-M_{H}^{2}\right)$ factor the $u$-channel $H$-exchange amplitudes are

$$
\begin{gathered}
M_{R R ; R R}^{H}(u)=-\frac{m_{l}^{2}}{E_{l}^{2}} y, \quad-M_{R R ; L R}^{H}(u)=M_{R R ; R L}^{H}=M_{R R ; L R}^{H}(t), \quad M_{R R ; L L}^{H}(u)=1-y, \\
M_{L R ; R R}^{H}(u)=M_{L R ; L L}^{H}(u)=M_{R R ; L R}^{H}(t) ; \quad M_{L R ; L R}^{H}(u)=M_{R R ; L L}^{H}(u), \quad M_{L R ; R L}^{H}(u)=M_{R R ; R R}^{H}(u), \\
M_{R L ; R R}^{H}(u)=-M_{R L ; L L}^{H}(u)=M_{R R ; L R}^{H}(t), \quad-M_{R L ; L R}^{H}(u)=M_{R R ; R R}^{H}(u), \quad M_{R L ; R L}^{H}(u)=M_{R R ; L L}^{H}(u), \\
M_{L L ; R R}^{H}(u)=M_{R R ; L L}^{H}(u), \quad M_{L L ; L R}^{H}(u)=M_{L L ; R L}^{H}(u)=M_{R R ; L R}^{H}(t), \quad M_{L L ; L L}^{H}(u)=-M_{R R ; R R}^{H}(u) .
\end{gathered}
$$

\section{APPENDIX B: TOTAL AMPLITUDES IN THE ESM}

We define

$$
M_{i j ; k l}=\sum_{X} M_{i j ; k l}^{X}(u)+\sum_{X} M_{i j ; k l}^{X}(t)+\sum_{X} M_{i j ; k l}^{X}(s)
$$

as the total amplitudes for fixed $i j ; k l$ polarization and exchanged particles $X=\gamma, Z^{0}, H^{0}$, and others. In the context of the standard model only $t$ and $u$ channels contribute. Notice also that these contributions arise only when the lepton-lepton scattering conserves flavors, $l_{1} l_{2} \rightarrow l_{1} l_{2}$ with $l_{1}$ being equal or not to $l_{2}$ :

$$
\begin{aligned}
M_{R R ; R R}= & i e^{2}\left\{\left(\frac{4 E^{2}-3 m_{l}^{2}}{2 E^{2}}\right)\left(\frac{u+t}{t u}\right)+\frac{m_{l}^{2}}{2 E^{2}}(1-2 y)\left(\frac{u-t}{t u}\right)+\frac{g^{2}}{e^{2}}\left(\frac{4 E^{2}-3 m_{l}^{2}}{2 E^{2}}\right)\left(g_{V}^{2}+g_{A}^{2}\right)\left[\frac{u+t-2 M_{Z}^{2}}{\left(t-M_{Z}^{2}\right)\left(u-M_{Z}^{2}\right)}\right]\right. \\
& -4 \frac{g^{2}}{e^{2}} g_{V} g_{A} \sqrt{\frac{E^{2}-m_{l}^{2}}{E^{2}}}\left[\frac{u+t-2 M_{Z}^{2}}{\left(t-M_{Z}^{2}\right)\left(u-M_{Z}^{2}\right)}\right]+\frac{g^{2}}{e^{2}} \frac{m_{l}^{2}}{2 E^{2}}(1-2 y)\left(g_{V}^{2}+g_{A}^{2}\right)\left[\frac{u-t}{\left(t-M_{Z}^{2}\right)\left(u-M_{Z}^{2}\right)}\right] \\
& \left.-\frac{m_{l}^{4}}{E^{2} e^{2} v^{2}}\left(\frac{1-y}{t-M_{H}^{2}}+\frac{y}{u-M_{H}^{2}}\right)\right\}, \\
M_{R R ; L R}= & i e^{2}\left[\frac{u+t}{t u}+\frac{g^{2}}{e^{2}} \frac{u-t}{\left(t-M_{Z}^{2}\right)\left(u-M_{Z}^{2}\right)}\left(g_{V}^{2}+g_{A}^{2}\right)+\frac{m_{l}^{2}}{e^{2} v^{2}} \frac{t-u}{\left(t-M_{H}^{2}\right)\left(u-M_{H}^{2}\right)}\right] \frac{m_{l}}{2 E} 2 y^{1 / 2}(1-2 y), \\
M_{R R ; R L}= & i e^{2}\left[\frac{u-t}{t u}+\frac{g^{2}}{e^{2}} \frac{u-t}{\left(t-M_{Z}^{2}\right)\left(u-M_{Z}^{2}\right)}\left(g_{V}^{2}-g_{A}^{2}\right)-\frac{m_{l}^{2}}{e^{2} v^{2}} \frac{t-u}{\left(t-M_{H}^{2}\right)\left(u-M_{H}^{2}\right)}\right] \frac{m_{l}}{2 E} 2 y^{1 / 2}(1-2 y),
\end{aligned}
$$




$$
\begin{aligned}
& M_{R R ; L L}=i e^{2}\left[\left(\frac{y}{t}+\frac{1-y}{u}\right) \frac{m_{l}^{2}}{E^{2}}+\frac{g^{2}}{e^{2}}\left(\frac{g_{V}^{2} y+g_{A}^{2}(4-2 y)}{t-M_{Z}^{2}}+\frac{g_{V}^{2}(1-y)-g_{A}^{2}(2+2 y)}{u-M_{Z}^{2}}\right) \frac{m_{l}^{2}}{2 E^{2}}+\frac{m_{l}^{2}}{e^{2} v^{2}}\left(\frac{y}{t-M_{H}^{2}}+\frac{1-y}{u-M_{H}^{2}}\right)\right], \\
& M_{L R ; R R}=i e^{2}\left[\frac{u-t}{t u}+\frac{g^{2}}{e^{2}} \frac{u+t-2 M_{Z}^{2}}{\left(t-M_{Z}^{2}\right)\left(u-M_{Z}^{2}\right)}\left(g_{V}^{2}-g_{A}^{2}\right)+\frac{m_{l}^{2}}{e^{2} v^{2}} \frac{u-t}{\left(t-M_{H}^{2}\right)\left(u-M_{H}^{2}\right)}\right] \frac{m_{l}}{2 E} 2 y^{1 / 2}(1-2 y), \\
& M_{L R ; L R}=i e^{2}\left[\frac{2}{t}-\frac{m_{l}^{2}}{E^{2}}\left(\frac{u-t}{t u}\right)+\frac{g^{2}}{e^{2}}\left[\frac{2}{t-M_{Z}^{2}}-\frac{m_{l}^{2}}{E^{2}}\left(\frac{u-t}{\left(t-M_{Z}^{2}\right)\left(u-M_{Z}^{2}\right)}\right)\right]\left(g_{V}^{2}-g_{A}^{2}\right)-\frac{m_{l}^{2}}{e^{2} v^{2}}\left(\frac{m_{l}^{2}}{E^{2}\left(t-M_{H}^{2}\right)}-\frac{1}{u-M_{H}^{2}}\right)\right](1-y), \\
& M_{L R ; R L}=i e^{2}\left[\frac{2}{u}-\frac{m_{l}^{2}}{E^{2}}\left(\frac{u-t}{t u}\right)+\frac{g^{2}}{e^{2}}\left[\frac{2}{u-M_{Z}^{2}}-\frac{m_{l}^{2}}{E^{2}}\left(\frac{t-u}{\left(t-M_{Z}^{2}\right)\left(u-M_{Z}^{2}\right)}\right)\right]\left(g_{V}^{2}-g_{A}^{2}\right)-\frac{m_{l}^{2}}{e^{2} v^{2}}\left(\frac{m_{l}^{2}}{E^{2}\left(u-M_{H}^{2}\right)}-\frac{1}{t-M_{H}^{2}}\right)\right] y \\
& M_{L R ; L L}=i e^{2}\left[\frac{u+t}{t u}+\frac{g^{2}}{e^{2}} \frac{u+t-2 M_{Z}^{2}}{\left(t-M_{Z}^{2}\right)\left(u-M_{Z}^{2}\right)}\left(g_{V}^{2}-g_{A}^{2}\right)-\frac{m_{l}^{2}}{e^{2} v^{2}} \frac{t+u-2 M_{H}^{2}}{\left(t-M_{H}^{2}\right)\left(u-M_{H}^{2}\right)}\right] \frac{m_{l}}{2 E} 2 y^{1 / 2}(1-2 y), \\
& M_{R L ; R R}=i e^{2}\left[\frac{u+t}{t u}+\frac{g^{2}}{e^{2}} \frac{u-t}{\left(t-M_{Z}^{2}\right)\left(u-M_{Z}^{2}\right)}\left(g_{V}^{2}-g_{A}^{2}\right)-\frac{m_{l}^{2}}{e^{2} v^{2}} \frac{t-u}{\left(t-M_{H}^{2}\right)\left(u-M_{H}^{2}\right)}\right] \frac{m_{l}}{2 E} 2 y^{1 / 2}(1-2 y), \\
& M_{R L ; L R}=-i e^{2}\left[\frac{-2}{u}+\frac{m_{l}^{2}}{E^{2}}\left(\frac{t-u}{t u}\right)-\frac{g^{2}}{e^{2}}\left[\frac{2}{u-M_{Z}^{2}}-\frac{m_{l}^{2}}{E^{2}}\left(\frac{t-u}{\left(t-M_{Z}^{2}\right)\left(u-M_{Z}^{2}\right)}\right)\right]\left(g_{V}^{2}-g_{A}^{2}\right)+\frac{m_{l}^{2}}{e^{2} v^{2}}\left(\frac{m_{l}^{2}}{E^{2}\left(u-M_{H}^{2}\right)}+\frac{1}{t-M_{H}^{2}}\right)\right] y \text {, } \\
& M_{R L ; R L}=i e^{2}\left[\frac{2}{t}-\frac{m_{l}^{2}}{E^{2}}\left(\frac{u-t}{t u}\right)+\frac{g^{2}}{e^{2}}\left[\frac{2}{t-M_{Z}^{2}}-\frac{m_{l}^{2}}{E^{2}}\left(\frac{u-t}{\left(t-M_{Z}^{2}\right)\left(u-M_{Z}^{2}\right)}\right)\right]\left(g_{V}^{2}-g_{A}^{2}\right)-\frac{m_{l}^{2}}{e^{2} v^{2}}\left(\frac{m_{l}^{2}}{E^{2}\left(t-M_{H}^{2}\right)}-\frac{1}{u-M_{H}^{2}}\right)\right](1-y), \\
& M_{R L ; L L}=i e^{2}\left[\frac{u-t}{u t}+\frac{g^{2}}{e^{2}} \frac{u+t-2 M_{Z}^{2}}{\left(t-M_{Z}^{2}\right)\left(u-M_{Z}^{2}\right)}\left(g_{V}^{2}-g_{A}^{2}\right)+\frac{m_{l}^{2}}{e^{2} v^{2}} \frac{t-u}{\left(t-M_{H}^{2}\right)\left(u-M_{H}^{2}\right)}\right] \frac{m_{l}}{2 E} 2 y^{1 / 2}(1-2 y), \\
& M_{L L ; R R}=i e^{2}\left[\left(\frac{y}{t}+\frac{1-y}{u}\right) \frac{m_{l}^{2}}{E^{2}}+\frac{g^{2}}{e^{2}}\left(\frac{g_{V}^{2} y+g_{A}^{2}(4-2 y)}{t-M_{Z}^{2}}+\frac{g_{V}^{2}(1-y)+g_{A}^{2}(2+2 y)}{u-M_{Z}^{2}}\right) \frac{m_{l}^{2}}{2 E^{2}}-\frac{m_{l}^{2}}{e^{2} v^{2}}\left(\frac{y}{t-M_{H}^{2}}-\frac{1-y}{u-M_{H}^{2}}\right)\right] \\
& M_{L L ; L R}=i e^{2}\left[\frac{t-u}{t u}+\frac{g^{2}}{e^{2}} \frac{u+t-2 M_{Z}^{2}}{\left(t-M_{Z}^{2}\right)\left(u-M_{Z}^{2}\right)}\left(g_{V}^{2}-g_{A}^{2}\right)-\frac{m_{l}^{2}}{e^{2} v^{2}} \frac{t-u}{\left(t-M_{H}^{2}\right)\left(u-M_{H}^{2}\right)}\right] \frac{m_{l}^{2}}{2 E} 2 y^{1 / 2}(1-2 y), \\
& M_{L L ; R L}=i e^{2}\left[\frac{u+t}{t u}+\frac{g^{2}}{e^{2}} \frac{u+t-2 M_{Z}^{2}}{\left(t-M_{Z}^{2}\right)\left(u-M_{Z}^{2}\right)}\left(g_{V}^{2}-g_{A}^{2}\right)-\frac{m_{l}^{2}}{e^{2} v^{2}} \frac{t-u}{\left(t-M_{H}^{2}\right)\left(u-M_{H}^{2}\right)}\right] \frac{m_{l}}{2 E} 2 y^{1 / 2}(1-2 y), \\
& M_{L L ; L L}=i e^{2}\left[\left(\frac{4 E^{2}-3 m_{l}^{2}}{2 E^{2}}\right)\left(\frac{u+t}{t u}\right)+\frac{m_{l}^{2}}{2 E^{2}}(1-2 y)\left(\frac{u-t}{t u}\right)+\frac{g^{2}}{e^{2}}\left(\frac{4 E^{2}-3 m_{l}^{2}}{2 E^{2}}\right)\left(g_{V}^{2}+g_{A}^{2}\right)\left(\frac{u+t-2 M_{Z}^{2}}{\left(t-M_{Z}^{2}\right)\left(u-M_{Z}^{2}\right)}\right)\right. \\
& +4 \frac{g^{2}}{e^{2}} g_{V} g_{A} \sqrt{\frac{E^{2}-m_{l}^{2}}{E^{2}}}\left(\frac{u+t-2 M_{Z}^{2}}{\left(t-M_{Z}^{2}\right)\left(u-M_{Z}^{2}\right)}\right)+\frac{g^{2}}{e^{2}} \frac{m_{l}^{2}}{2 E^{2}}(1-2 y)\left(g_{V}^{2}+g_{A}^{2}\right)\left(\frac{u-t}{\left(t-M_{Z}^{2}\right)\left(u-M_{Z}^{2}\right)}\right) \\
& \left.+\frac{m_{l}^{4}}{E^{2} e^{2} v^{2}}\left(\frac{1-y}{t-M_{H}^{2}}+\frac{y}{u-M_{H}^{2}}\right)\right]
\end{aligned}
$$




\section{APPENDIX C: DIFFERENTIAL CROSS SECTION FOR FIXED TARGETS AND COLLIDERS IN THE ESM}

In the context of the standard model in a collider experiment we have

$$
\begin{aligned}
\left.\frac{d \sigma^{\mathrm{ESM}}}{d \Omega}\right|_{\mathrm{CO}}= & \frac{e^{4}}{128 \pi^{2} s}\left\{\frac{1+y^{4}+(1-y)^{4}}{y^{2}(1-y)^{2}}+\frac{2 g^{2}}{e^{2}}\left[\left(g_{V}^{2}+g_{A}^{2}\right) \frac{1+2 a}{y(1-y)(y+a)[1-y+a]}+\left(g_{V}^{2}-g_{A}^{2}\right)\right.\right. \\
& \left.\times\left(\frac{(1-y)^{2}}{y(y+a)}+\frac{y^{2}}{(1-y)[1-y+a]}\right)\right]+\frac{g^{4}}{e^{4}}\left(g_{V}^{2}-g_{A}\right)^{2}\left(\frac{(1-y)^{2}}{(y+a)^{2}}+\frac{y^{2}}{(1-y+a)^{2}}\right) \\
& \left.+\frac{g^{4}}{e^{4}}\left(g_{V}^{4}+6 g_{V}^{2} g_{A}^{2}+g_{A}^{4}\right) \frac{(1+2 a)^{2}}{(y+a)^{2}[1-y+a]}\right\},
\end{aligned}
$$

where $a \equiv M_{Z}^{2} / s$.

For the fixed target experiment we have

$$
\left.\frac{d \sigma^{\mathrm{ESM}}}{d \Omega}\right|_{\mathrm{FT}} \approx \frac{e^{4}}{128 \pi^{2} m_{e} p_{e}} \frac{p_{e^{\prime}}^{2}}{\left(E_{e}+m_{e}\right) p_{e^{\prime}}-p_{e} E_{e^{\prime}} \cos \theta_{e e^{\prime}}}\left[\frac{1+y+(1-y)^{4}}{y^{2}(1-y)^{2}}\right],
$$

where we have written only the main contribution. Here $p_{e}\left(E_{e}\right)$ and $p_{e^{\prime}}\left(E_{e^{\prime}}\right)$ denote the momentum (energy) of the initial and final electron, respectively; $\cos \theta_{e e^{\prime}}$ is the cosine of the angle between the incident electron and one of the final electrons.

\section{APPENDIX D: $U$ AMPLITUDES}

In this appendix we give the invariant amplitudes for $l l \rightarrow l^{\prime} l^{\prime}$ scattering in the context of a model with a doubly charged vector bilepton $U^{--}$. In principle, the Higgs contributions are not negligible. However, for the sake of simplicity we will not take the extra scalar contributions into account.

Up to a common $\mathrm{ig}^{2} / 2\left(s-M_{U}^{2}\right)$ factor, the $s$-channel $U^{--}$-exchange amplitudes are

$$
\begin{aligned}
& M_{R R ; R R}^{U}(s)=0, \quad M_{R R ; L R}^{U}(s)=0, \quad M_{R R ; R L}^{U}(s)=0, \quad M_{R R ; L L}^{U}(s)=0, \\
& M_{L R ; R R}^{U}(s)=-\frac{1}{2}\left(\frac{E_{l}-m_{l}}{E_{l}} \frac{E_{l}^{\prime}-m_{l}^{\prime}}{E_{l}^{\prime}}\right)^{1 / 2}\left[1-\left(\frac{E_{l}+m_{l}}{E_{l}-m_{l}} \frac{E_{l}^{\prime}+m_{l}^{\prime}}{E_{l}^{\prime}-m_{l}^{\prime}}\right)^{1 / 2}\right] 2 y^{1 / 2}(1-2 y), \\
& M_{L R ; L R}^{U}(s)=\left(\frac{E_{l}-m_{l}}{E_{l}} \frac{E_{l}^{\prime}-m_{l}^{\prime}}{E_{l}^{\prime}}\right)^{1 / 2}\left[1+\left(\frac{E_{l}+m_{l}}{E_{l}-m_{l}} \frac{E_{l}^{\prime}+m_{l}^{\prime}}{E_{l}^{\prime}-m_{l}^{\prime}}\right)^{1 / 2}\right](1-y), \\
& M_{L R ; R L}^{U}(s)=-\left(\frac{E_{l}-m_{l}}{E_{l}} \frac{E_{l}^{\prime}-m_{l}^{\prime}}{E_{l}^{\prime}}\right)^{1 / 2}\left[1-\left(\frac{E_{l}+m_{l}}{E_{l}-m_{l}} \frac{E_{l}^{\prime}+m_{l}^{\prime}}{E_{l}^{\prime}-m_{l}^{\prime}}\right)^{1 / 2}\right] y, \\
& M_{L R ; L L}^{U}(s)=-\frac{1}{2}\left(\frac{E_{l}-m_{l}}{E_{l}} \frac{E_{l}^{\prime}-m_{l}^{\prime}}{E_{l}^{\prime}}\right)^{1 / 2}\left[1-\left(\frac{E_{l}+m_{l}}{E_{l}-m_{l}} \frac{E_{l}^{\prime}+m_{l}^{\prime}}{E_{l}^{\prime}-m_{l}^{\prime}}\right)^{1 / 2}\right] 2 y^{1 / 2}(1-2 y) . \\
& M_{R L ; R R}^{U}(s)=-\frac{1}{2}\left(\frac{E_{l}-m_{l}}{E_{l}} \frac{E_{l}^{\prime}-m_{l}^{\prime}}{E_{l}^{\prime}}\right)^{1 / 2}\left[1-\left(\frac{E_{l}+m_{l}}{E_{l}-m_{l}} \frac{E_{l}^{\prime}+m_{l}^{\prime}}{E_{l}^{\prime}-m_{l}^{\prime}}\right)^{1 / 2}\right] 2 y^{1 / 2}(1-2 y), \\
& M_{R L ; L R}^{U}(s)=\left(\frac{E_{l}-m_{l}}{E_{l}} \frac{E_{l}^{\prime}-m_{l}^{\prime}}{E_{l}^{\prime}}\right)^{1 / 2}\left[1+\left(\frac{E_{l}+m_{l}}{E_{l}-m_{l}} \frac{E_{l}^{\prime}+m_{l}^{\prime}}{E_{l}^{\prime}-m_{l}^{\prime}}\right)^{1 / 2}\right] y, \\
& M_{R L ; R L}^{U}(s)=-\left(\frac{E_{l}-m_{l}}{E_{l}} \frac{E_{l}^{\prime}-m_{l}^{\prime}}{E_{l}^{\prime}}\right)^{1 / 2}\left[1+\left(\frac{E_{l}+m_{l}}{E_{l}-m_{l}} \frac{E_{l}^{\prime}+m_{l}^{\prime}}{E_{l}^{\prime}-m_{l}^{\prime}}\right)^{1 / 2}\right] y,
\end{aligned}
$$




$$
\begin{gathered}
M_{R L ; L L}^{U}(s)=\frac{1}{2}\left(\frac{E_{l}-m_{l}}{E_{l}} \frac{E_{l}^{\prime}-m_{l}^{\prime}}{E_{l}^{\prime}}\right)^{1 / 2}\left[1-\left(\frac{E_{l}+m_{l}}{E_{l}-m_{l}} \frac{E_{l}^{\prime}+m_{l}^{\prime}}{E_{l}^{\prime}-m_{l}^{\prime}}\right)^{1 / 2}\right] 2 y^{1 / 2}(1-2 y), \\
M_{L L ; R R}^{U}(s)=0, \quad M_{L L ; L R}^{U}(s)=0, \quad M_{L L ; R L}^{U}(s)=0, \quad M_{L L ; L L}^{U}(s)=0, \\
M_{R R}^{U}(s)=M_{R R ; R R}^{U}(s)+M_{R R ; L R}^{U}(s)+M_{R R ; R L}^{U}(s)+M_{R R ; L L}^{U}(s)=0, \\
M_{R L}^{U}(s)=M_{R L ; R R}^{U}(s)+M_{R L ; L R}^{U}(s)+M_{R L ; R L}^{U}(s)+M_{R L ; L L}^{U}(s) \\
=\frac{i g^{2}}{2\left(s-M_{U}^{2}\right)}\left(\frac{E_{l}-m_{l}}{E_{l}} \frac{E_{l}^{\prime}-m_{l}^{\prime}}{E_{l}^{\prime}}\right)^{1 / 2}\left[1+\left(\frac{E_{l}+m_{l}}{E_{l}-m_{l}} \frac{E_{l}^{\prime}+m_{l}^{\prime}}{E_{l}^{\prime}-m_{l}^{\prime}}\right)^{1 / 2}\right](1-2 y), \\
M_{L R}^{U}(s)=M_{L R ; R R}^{U}(s)+M_{L R ; L R}^{U}(s)+M_{L R ; R L}^{U}(s)+M_{L R ; L L}^{U}(s) \\
=\frac{i g^{2}}{2\left(s-M_{U}^{2}\right)}\left(\frac{E_{l}-m_{l}}{E_{l}} \frac{E_{l}^{\prime}-m_{l}^{\prime}}{E_{l}^{\prime}}\right)^{1 / 2}\left[1+\left(\frac{E_{l}+m_{l}}{E_{l}-m_{l}} \frac{E_{l}^{\prime}+m_{l}^{\prime}}{E_{l}^{\prime}-m_{l}^{\prime}}\right)^{1 / 2}\right](1-2 y), \\
M_{L L}^{U}(s)=M_{L L ; R R}^{U}(s)+M_{L L ; L R}^{U}(s)+M_{L L ; R L}^{U}(s)+M_{L L ; L L}^{U}(s)=0 .
\end{gathered}
$$

For the collider case, if there exists a doubly charged vector boson $U^{--}$which is not too heavy, it will appear at the energies of the next linear colliders (for electron beams up to $1.5 \mathrm{TeV}$ ). In this case the cross section at the $U$ peak will be larger than in the case of the standard model. Explicitly, the collider cross section in the case of a $U$ exchange besides the ESM contributions is

$$
\begin{aligned}
\left.\frac{d \sigma^{\mathrm{ESM}+\mathrm{U}}}{d \Omega}\right|_{\mathrm{CO}}= & \left.\frac{d \sigma^{\mathrm{ESM}}}{d \Omega}\right|_{\mathrm{CO}}+\frac{e^{4}}{128 \pi^{2} s}\left[\frac{g^{2}}{4 e^{4}(1-b)^{2}}\left[(1-y)^{2}+y^{2}\right]+\frac{g^{2}}{e^{2}(1-b)}\right. \\
& \left.\times\left(\frac{y^{3}-(1-y)^{3}}{y(1-y)}+\frac{g^{2}}{e^{2}}\left(g_{V}^{2}-g_{A}^{2}\right) \frac{y^{2}(y+a)-(1-y)^{2}(1-y+a)}{(y+a)(1-y+a)}\right)\right],
\end{aligned}
$$

where $b=M_{U}^{2} / s$.

\section{APPENDIX E: $\boldsymbol{H}^{++}$AMPLITUDES}

Here we will consider the amplitudes of the two doubly charged scalars $H_{1}^{++}$and $H_{2}^{++}$which are present in the model of Ref. [12]. The former is part of a triplet of $\mathrm{SU}(2)$ and the latter one is a singlet of $\mathrm{SU}(2)$. Hence, the experimental lower bound on their masses will be different. Up to a common $2 m_{l}^{2}\left(m_{l}-E\right)^{2} / v_{S}^{2}\left(s-M_{H_{i}}^{2}\right), \quad i=1,2$, factor the amplitudes are

$$
M_{R R ; R R}^{H_{i}}=-M_{R R ; L L}^{H_{i}}=M_{L L ; L L}^{H_{i}}=-M_{L L ; R R}^{H_{i}}=\frac{\left(m_{l}-E\right)\left(E+m_{l}\right)}{E^{2}+m_{l}^{2}},
$$

with the other amplitudes vanishing.

[1] The NLC Accelerator Design Group and The NLC Physics Working Group, S. Kuhman et al., "Physics and Technology of the Next Linear Collider," Report No. FERMILABPUB96/112. See also http://nlc.physics.upenn.edu/nlc/nlc.html and http://pss058.psi.ch/cuypers/e-e-.htm.

[2] J. F. Gunion, "Muon Colliders: The Machine and the Physics," hep-ph/9707379. See also http//www.cap.bnl.gov/mumu and http://www.fnal.gov/projects/muon_collider.

[3] E. Derman and W. Marciano, Ann. Phys. (N.Y.) 121, 147 (1979).
[4] S. L. Glashow, Nucl. Phys. 22, 579 (1961); S. Weinberg, Phys. Rev. Lett. 19, 1264 (1967); A. Salam, in Elementary Particle Theory; Relativistic Groups and Analyticity (Nobel Symposium No. 8), edited by N. Svartholm (Almqvist and Wiksell, Stockholm, 1968).

[5] See htpp://www.salc.stanford.edu/FIND/explist.html.

[6] W. C. Barbert, G. K. O'Neil, R. Gittelman, and B. Richter, Phys. Rev. D 3, 2796 (1971).

[7] A. Czarnecki and W. Marciano, Phys. Rev. D 53, 1066 (1996).

[8] A. M. Bincer, Phys. Rev. 107, 1434 (1957). 
[9] P. S. Cooper et al., Phys. Rev. Lett. 34, 1589 (1975).

[10] N. M. Sumeiko and J. G. Suarez, talk given at the International School on Actual Problems of Particle Physics, Gmel, Belarus, 1997, hep-ph/9712407.

[11] Recently the fact that the left-right asymmetry is large in colliders has been pointed out by A. Czarnecki and W. Marciano, Int. J. Mod. Phys. A 13, 2235 (1998).

[12] F. Pisano and V. Pleitez, Phys. Rev. D 46, 410 (1992).

[13] J. T. Liu and D. Ng, Phys. Rev. D 50, 548 (1994).

[14] F. Cuypers and M. Raidal, Nucl. Phys. B501, 3 (1997).

[15] Particle Data Group, R. M. Barnett et al., Phys. Rev. D 54,
1 (1996).

[16] OPAL Collaboration, P. D. Acton et al., Phys. Lett. B 295, 347 (1992).

[17] M. Swartz et al., Phys. Rev. Lett. 64, 2877 (1995).

[18] J. F. Gunion, C. Loomis, and K. T. Pitts, in Proceedings of 1996 DPF/DPB Summer Study on New Directions for HighEnergy Physics (Snowmass 96), Snowmass, Colorado, 1996, edited by D. G. Cassel, L. Trindle Gennari, and R. H. Siemann (SLAC, Stanford, 1997), hep-ph/9610237.

[19] F. Cuypers and S. Davidson, Eur. Phys. J. C2, 503 (1998), and references therein. 